\title{
On the Well-Posedness of the Boussinesq Equations with Anisotropic Filter for Turbulent Flows
}

\author{
Luigi C. Berselli and Davide Catania
}

\begin{abstract}
We consider approximate deconvolution models for the Boussinesq equations, based on suitable anisotropic filters. We discuss existence and well-posedness of the solutions, with particular emphasis on the role of the energy (of the model) balance.
\end{abstract}

Keywords. Boussinesq equations, anisotropic filters, Large Eddy Simulation (LES), turbulent flows in domains with boundary, approximate deconvolution methods

Mathematics Subject Classification (2010). Primary 76D05, secondary 35Q30, 76F65, 76D03

\section{Introduction}

In this paper we make another step in the direction of designing robust and reliable tools for the study of mixing phenomena, with possible applications to modeling of certain geophysical flows as for instance oceanic flow models [24] or volcanic plumes [14] (at least in certain regimes). We wish to develop a complete new family of large scale methods to simulate mixing and to perform numerical simulations of the Oberbeck-Boussinesq equations (which are generally simply known as Boussinesq equations, see Rajagopal, Růžička, and Srinivasa [26]), when Direct Numerical Simulations (DNS) are not feasible. In particular, the numerical simulation of fluids which can be described by the Boussinesq equations, as explained in Majda [23] and Cushman-Roisin and Beckers [17], presents - at least- the same difficulties of the Navier-Stokes equations, and the range of active scales precludes a DNS in a foreseeable future, see for instance Sagaut [28], Geurts [21], and also [4,6]. Moreover, for the Boussinesq equations,

L. C. Berselli: Dipartimento di Matematica, Università di Pisa, Via F. Buonarroti 1/c, 56125 Pisa, Italy; berselli@dma.unipi.it

D. Catania: DICATAM, Sezione Matematica, Università di Brescia, Via Valotti 9, 25133 Brescia, Italy; davide.catania@unibs.it. 
a phenomenology à la Kolmogorov with a quantitative description involving eddies decaying towards smaller and smaller scales is not clear. Hence, one can try to collect relevant modeling properties from other sources.

In this paper (after passing to suitable non-dimensional quantities and with positive constants $\nu$ and $K$ ) we consider the Boussinesq equations

$$
\begin{aligned}
\partial_{t} \boldsymbol{u}+\nabla \cdot(\boldsymbol{u} \otimes \boldsymbol{u})-\nu \Delta \boldsymbol{u}+\nabla \pi & =-\vartheta \boldsymbol{e}_{3}, \\
\nabla \cdot \boldsymbol{u} & =0, \\
\partial_{t} \vartheta+\nabla \cdot(\vartheta \boldsymbol{u})-K \Delta \vartheta & =0,
\end{aligned}
$$

and especially the filtered counterpart, which can be used to model turbulent stratified flows. We will consider the Boussinesq equations in a bounded domain and in presence of Dirichlet boundary conditions. In particular, we continue the theoretical investigation on Large Eddy Simulation (LES) models applied to stratified flows, for which recent numerical results are reported in $[3,24$, 25]. By adapting and extending ideas from [1], where only the Navier-Stokes equations have been considered, we construct different families of Large Eddy Simulation models based on differential filters and zeroth order deconvolution, and we analyze the well-posedness of the corresponding initial boundary value problems.

To emphasize the relevance of the analytical results that we obtain, we observe that most of the results (existence, uniqueness, ... ) valid for the NavierStokes equations are still valid also for the Boussinesq equations $[8,19]$. On the other hand, we stress that previous results on the Navier-Stokes equations and anisotropic filters as those in [1] use in a peculiar manner the interchangeable order of the convection/convected-field in the nonlinear term of the NavierStokes equations, and the additional regularity coming form the divergence-free constraint [15]. This is not anymore true in the transport equation involving the variable $\rho$ (which corresponds to the variation of density $\vartheta$ ) and the adaption of some results using also anisotropic regularity of the two different fields is not straightforward, hence deserves some analysis.

As explained step-by-step in [1] (hence we skip full details here), we use a setting in which we can define in a proper way the filtering, even in presence of boundaries, using a channel-type domain. Recall just that the idea of anisotropic filters comes back from the seventies and the work of Deardorff, but in the context of Approximate Deconvolution LES Models (ADM), the most important reference is the paper by Stolz, Adams, and Kleiser [29], from which we have borrowed and extended some ideas. Concerning Approximate deconvolution, we consider mainly the zeroth order approximation, with some variants in the filtering, as in Layton and Lewandowski [22]. Recently, in [9], the asymptotic behavior in terms of the order of deconvolution (proved for the Navier-Stokes equations in [7] and for magnetohydrodynamics in [2]) have been adapted to the Boussinesq system with periodic boundary conditions. 
A special role is played by a suitable splitting of horizontal and vertical unknowns and variables and we will use the following notation:

$$
\begin{gathered}
\boldsymbol{x}:=\left(x_{1}, x_{2}, x_{3}\right), \quad \boldsymbol{x}_{\mathrm{h}}:=\left(x_{1}, x_{2}\right), \\
\partial_{j}:=\partial_{x_{j}}, \quad \Delta_{\mathrm{h}}:=\partial_{1}^{2}+\partial_{2}^{2}, \quad \nabla_{\mathrm{h}}:=\left(\partial_{1}, \partial_{2}\right),
\end{gathered}
$$

where "h" stays for "horizontal". For a vector function $\boldsymbol{w}:=\left(w^{1}, w^{2}, w^{3}\right)$, we set $\boldsymbol{w}^{\mathrm{h}}:=\left(w^{1}, w^{2}\right)$. The physical domain is the following flat channel

$$
D:=\left\{\boldsymbol{x} \in \mathbb{R}^{3}:-\pi<x_{1}, x_{2}<\pi,-d<x_{3}<d\right\},
$$

and we impose $2 \pi$-periodicity with respect to $\boldsymbol{x}_{\mathrm{h}}$ (i.e. with respect to both $x_{1}$ and $x_{2}$ ) and homogeneous Dirichlet conditions on the boundary

$$
\Gamma:=\left\{\boldsymbol{x} \in \mathbb{R}^{3}:-\pi<x_{1}, x_{2}<\pi, x_{3}= \pm d\right\} .
$$

We also observe that one main geometric properties is that the boundary is flat. (This is not unreasonable, since in oceanic flows horizontal scales are much larger than the vertical as in the primitive equations $[10,11]$ or in the equations for horizontal Ekman layers [15].) We introduce the following function spaces:

$$
\begin{aligned}
L^{2}(D) & :=\left\{\phi: D \rightarrow \mathbb{R} \text { measurable, } 2 \pi \text { periodic in } x_{\mathrm{h}}, \int_{D}|\phi|^{2} \mathrm{~d} \boldsymbol{x}<+\infty\right\}, \\
L_{0}^{2}(D) & :=\left\{\phi \in L^{2}(D) \text { with zero mean with respect to } \boldsymbol{x}_{\mathrm{h}}\right\}, \\
H & :=\left\{\boldsymbol{\phi} \in\left(L_{0}^{2}(D)\right)^{3}: \nabla \cdot \boldsymbol{\phi}=0 \text { in } D, \boldsymbol{\phi} \cdot \boldsymbol{n}=0 \text { on } \Gamma\right\},
\end{aligned}
$$

( $\boldsymbol{n}$ is the outward normal to $\Gamma$ ), all with $L^{2}$ norm denoted by $\|\cdot\|$, and $L^{2}$ scalar product $(\cdot, \cdot)$. Here and in the following, we use the same notation for scalar and vector valued functions, when they make sense. Moreover, we also set

$$
\begin{aligned}
H_{\mathrm{h}}^{1} & :=\left\{\phi \in L_{0}^{2}(D): \nabla_{\mathrm{h}} \phi \in\left(L^{2}(D)\right)^{2}\right\}, \\
V_{\mathrm{h}} & :=\left\{\boldsymbol{\phi} \in H: \nabla_{\mathrm{h}} \boldsymbol{\phi} \in\left(L^{2}(D)\right)^{6}\right\}, \\
V & :=\left\{\boldsymbol{\phi} \in H: \nabla \boldsymbol{\phi} \in\left(L^{2}(D)\right)^{9} \text { and } \boldsymbol{\phi}=\mathbf{0} \text { on } \Gamma\right\}, \\
H_{\mathrm{h}}^{2} & :=\left\{\phi \in H_{\mathrm{h}}^{1}: \nabla_{\mathrm{h}} \nabla \phi \in\left(L^{2}(D)\right)^{6}\right\},
\end{aligned}
$$

and denote by $V^{*}$ the topological dual space to $V$. We denote by $L^{p}$ and $H^{m}$ classical Lebesgue and Sobolev spaces. When dealing with the Bochner space $L^{p}(0, T ; X)$ we use the short notation $\|\cdot\|_{L^{p} X}$ for its norm. Weakly continuous functions are denoted by the symbol $C_{\mathrm{w}}$.

A main mathematical limitation to introduce ADM in presence of solid boundaries is related with the boundary conditions to be imposed on the operator used for filtering. The lack of commutation between differentiation and 
filtering (cf. $[5,18])$ is source of problems in deriving the filtered equations. To this end, we introduce the anisotropic (horizontal) filter

$$
\overline{\boldsymbol{v}}^{\mathrm{h}}=A_{\mathrm{h}}^{-1} \boldsymbol{v}, \quad \text { where } A_{\mathrm{h}}:=I-\alpha^{2} \Delta_{\mathrm{h}},
$$

(with periodic conditions in $\boldsymbol{x}_{\mathrm{h}}$ ). Let us note that the filter is linear and commutes with differentiation with respect to the vector variable $\boldsymbol{x}$.

\section{A doubly filtered LES model}

We first start considering a double filtered model, that is a model in which both equations are filtered by applying the operator $A_{\mathrm{h}}^{-1}$. Filtering the equations for $\boldsymbol{u}$ and the equation for $\vartheta$, we obtain

$$
\begin{aligned}
\partial_{t} \overline{\boldsymbol{u}}^{\mathrm{h}}+\nabla \cdot \overline{(\boldsymbol{u} \otimes \boldsymbol{u}}^{\mathrm{h}}-\nu \Delta \overline{\boldsymbol{u}}^{\mathrm{h}}+\nabla \bar{\pi}^{\mathrm{h}} & =-\bar{\vartheta}^{\mathrm{h}} \boldsymbol{e}_{3}, \\
\nabla \cdot \overline{\boldsymbol{u}}^{\mathrm{h}} & =0, \\
\partial_{t} \bar{\vartheta}^{\mathrm{h}}+\nabla \cdot \overline{(\vartheta \boldsymbol{u})^{\mathrm{h}}}-K \Delta \bar{\vartheta}^{\mathrm{h}}=0 . &
\end{aligned}
$$

We set as usual $\boldsymbol{w}:=\overline{\boldsymbol{u}}^{\mathrm{h}}, q:=\bar{\pi}^{\mathrm{h}}$ and $\rho:=\bar{\vartheta}^{\mathrm{h}}$, so that $\boldsymbol{u}=A_{\mathrm{h}} \boldsymbol{w}$ and $\vartheta=A_{\mathrm{h}} \rho$. We then address the interior closure problem by the zeroth order approximations

$$
\begin{aligned}
& \overline{\boldsymbol{u} \otimes \boldsymbol{u}}^{\mathrm{h}} \approx{\overline{\overline{\boldsymbol{u}}^{\mathrm{h}} \otimes \overline{\boldsymbol{u}}^{\mathrm{h}}}}^{\mathrm{h}}=\overline{\boldsymbol{w} \otimes \boldsymbol{w}^{\mathrm{h}}}, \\
& \overline{\vartheta \boldsymbol{u}} \approx \overline{\bar{\vartheta}}^{\mathrm{h}} \overline{\boldsymbol{u}}^{\mathrm{h}} \approx \bar{\rho} \overline{\boldsymbol{w}}^{\mathrm{h}},
\end{aligned}
$$

so that we finally deduce the approximate model

$$
\begin{aligned}
& \left.\partial_{t} \boldsymbol{w}+\nabla \cdot \overline{(\boldsymbol{w} \otimes \boldsymbol{w}}^{\mathrm{h}}-\nu \Delta \boldsymbol{w}+\nabla q=-\rho \boldsymbol{e}_{3} \quad \text { in } D \times\right] 0, T[, \\
& \nabla \cdot \boldsymbol{w}=0 \quad \text { in } D \times] 0, T[, \\
& \left.\partial_{t} \rho+\nabla \cdot \overline{(\rho \boldsymbol{w})}^{\mathrm{h}}-K \Delta \rho=0 \quad \text { in } D \times\right] 0, T[.
\end{aligned}
$$

Remark 2.1. It is possible to use different filters for $\boldsymbol{u}$ and $\vartheta$. In particular, we can introduce the filters

$$
\overline{\boldsymbol{v}}^{\alpha_{i}}:=A_{i}^{-1} \boldsymbol{v} \quad \text { with } A_{i}:=I-\alpha_{i}^{2} \Delta_{\mathrm{h}} \text {, where } \alpha_{i}>0 \text { for } i=1,2,
$$

and use $A_{1}$ in the equations for $\boldsymbol{u}$ and $A_{2}$ in the equation for $\vartheta$. Setting $\boldsymbol{w}=\overline{\boldsymbol{u}}^{\alpha_{1}}$, $q=\bar{\pi}^{\alpha_{1}}$ and $\rho=\bar{\vartheta}^{\alpha_{2}}$, we deduce

$$
\begin{aligned}
\partial_{t} \boldsymbol{w}+\nabla \cdot \overline{(\boldsymbol{w} \otimes \boldsymbol{w}}^{\alpha_{1}}-\nu \Delta \boldsymbol{w}+\nabla q & =-A_{2} \bar{\rho}^{\alpha_{1}} \boldsymbol{e}_{3}, \\
\nabla \cdot \boldsymbol{w} & =0, \\
\partial_{t} \rho+\nabla \cdot \overline{(\rho \boldsymbol{w})}^{\alpha_{2}}-K \Delta \rho & =0 .
\end{aligned}
$$

The regularity of the solutions does not change, and the proof can be reproduced straightforwardly. We choose to use just one filter to simplify the notation and keep the proofs more easily readable. 
In order to make the equations (1)-(3) have a meaning, let us assume $\boldsymbol{w}(t): D \rightarrow \mathbb{R}^{3}$ and $\rho(t): D \rightarrow \mathbb{R}$. We say that $(\boldsymbol{w}, \rho)$ is a regular weak solution (omitting the pressure term q) to (1)-(3), with $\boldsymbol{w}=\mathbf{0}$ and $\rho=0$ on ] $0, T\left[\times \Gamma\right.$, and $(\boldsymbol{w}(0, \boldsymbol{x}), \rho(0, \boldsymbol{x}))=\left(\boldsymbol{w}_{0}, \rho_{0}\right) \in V_{\mathrm{h}} \times H_{\mathrm{h}}^{1}$, when the following properties are verified.

- Regularity:

$$
\begin{aligned}
\boldsymbol{w} & \in L^{\infty}\left(0, T ; V_{\mathrm{h}}\right) \cap L^{2}\left(0, T ; V \cap\left(H_{\mathrm{h}}^{2}\right)^{3}\right) \cap C_{\mathrm{w}}\left(0, T ; V_{\mathrm{h}}\right), \\
w^{3} & \in L^{\infty}\left(0, T ; H^{1}\right) \cap L^{2}\left(0, T ; H^{2}\right), \\
\partial_{t} \boldsymbol{w} & \in L^{2}\left(0, T ; V^{*}\right), \\
\rho & \in L^{\infty}\left(0, T ; H_{\mathrm{h}}^{1}\right) \cap L^{2}\left(0, T ; H^{1} \cap H_{\mathrm{h}}^{2}\right) \cap C_{\mathrm{w}}\left(0, T ; H_{\mathrm{h}}^{1}\right), \\
\partial_{t} \rho & \in L^{2}\left(0, T ; H^{-1}\right) .
\end{aligned}
$$

- Weak formulation:

$$
\begin{aligned}
& \int_{0}^{+\infty}\left\{\left(\boldsymbol{w}, \partial_{t} \boldsymbol{\varphi}\right)-\nu(\nabla \boldsymbol{w}, \nabla \boldsymbol{\varphi})+\left(\boldsymbol{w} \otimes \boldsymbol{w}, \nabla \overline{\boldsymbol{\varphi}}^{\mathrm{h}}\right)\right\}(s) \mathrm{d} s \\
& =\int_{0}^{+\infty}\left(\rho \boldsymbol{e}_{3}, \boldsymbol{\varphi}\right)(s) \mathrm{d} s-(\boldsymbol{w}(0), \boldsymbol{\varphi}(0)), \\
& \int_{0}^{+\infty}\left\{\left(\rho, \partial_{t} \psi\right)+\left(\rho \boldsymbol{w}, \nabla \bar{\psi}^{\mathrm{h}}\right)-K(\nabla \rho, \nabla \psi)\right\}(s) \mathrm{d} s \\
& =-(\rho(0), \psi(0)),
\end{aligned}
$$

for each $\varphi \in\left(C_{0}^{\infty}\left(D \times[0, T[))^{3}\right.\right.$ such that $\nabla \cdot \varphi=0$, and for each $\psi \in$ $C_{0}^{\infty}(D \times[0, T[)$ or, equivalently (see $[20])$,

$$
\begin{aligned}
& \int_{t_{0}}^{t_{1}}\left\{\left(\boldsymbol{w}, \partial_{t} \boldsymbol{\varphi}\right)-\nu(\nabla \boldsymbol{w}, \nabla \boldsymbol{\varphi})+\left(\boldsymbol{w} \otimes \boldsymbol{w}, \nabla \overline{\boldsymbol{\varphi}}^{\mathrm{h}}\right)\right\}(s) \mathrm{d} s \\
& =\int_{t_{0}}^{t_{1}}\left(\rho \boldsymbol{e}_{3}, \boldsymbol{\varphi}\right)(s) \mathrm{d} s+\left(\boldsymbol{w}\left(t_{1}\right), \boldsymbol{\varphi}\left(t_{1}\right)\right)-\left(\boldsymbol{w}\left(t_{0}\right), \boldsymbol{\varphi}\left(t_{0}\right)\right), \\
& \int_{t_{0}}^{t_{1}}\left\{\left(\rho, \partial_{t} \psi\right)+\left(\rho \boldsymbol{w}, \nabla \bar{\psi}^{\mathrm{h}}\right)-K(\nabla \rho, \nabla \psi)\right\}(s) \mathrm{d} s \\
& =\left(\rho\left(t_{1}\right), \psi\left(t_{1}\right)\right)-\left(\rho\left(t_{0}\right), \psi\left(t_{0}\right)\right),
\end{aligned}
$$

for each $0 \leq t_{0} \leq t_{1}<T$.

The main theorem of this section is the following existence and uniqueness result. 
Theorem 2.2. Let be given $\left(\boldsymbol{w}_{0}, \rho_{0}\right) \in V_{\mathrm{h}} \times H_{\mathrm{h}}^{1}$ and $\nu, K>0$. Then, there exists a unique regular weak solution to (1)-(3), with $\boldsymbol{w}=\mathbf{0}$ and $\rho=0$ on ] $0, T\left[\times \Gamma\right.$, and $(\boldsymbol{w}(0, \boldsymbol{x}), \rho(0, \boldsymbol{x}))=\left(\boldsymbol{w}_{0}, \rho_{0}\right)$, depending continuously on the data (the system is well-posed). Moreover, the regular weak solution satisfies the energy (of the model) identity

$$
\begin{aligned}
& \frac{1}{2}\left(\|\boldsymbol{w}(t)\|^{2}+\alpha^{2}\left\|\nabla_{\mathrm{h}} \boldsymbol{w}(t)\right\|^{2}+\|\rho(t)\|^{2}+\alpha^{2}\left\|\nabla_{\mathrm{h}} \rho(t)\right\|^{2}\right) \\
& \quad+\nu \int_{0}^{t}\left(\|\nabla \boldsymbol{w}(s)\|^{2}+\alpha^{2}\left\|\nabla_{\mathrm{h}} \nabla \boldsymbol{w}(s)\right\|^{2}\right) \mathrm{d} s+K \int_{0}^{t}\left(\|\nabla \rho(s)\|^{2}+\alpha^{2}\left\|\nabla_{\mathrm{h}} \nabla \rho(s)\right\|^{2}\right) \mathrm{d} s \\
& =\frac{1}{2}\left(\|\boldsymbol{w}(0)\|^{2}+\alpha^{2}\left\|\nabla_{\mathrm{h}} \boldsymbol{w}(0)\right\|^{2}+\|\rho(0)\|^{2}+\alpha^{2}\left\|\nabla_{\mathrm{h}} \rho(0)\right\|^{2}\right) \\
& \quad-\int_{0}^{t}\left(A_{\mathrm{h}}^{\frac{1}{2}} \rho(s) \boldsymbol{e}_{3}, A_{\mathrm{h}}^{\frac{1}{2}} \boldsymbol{w}(s)\right) \mathrm{d} s .
\end{aligned}
$$

Proof. We divide the proof in three parts: first we prove existence and energy inequality, then we show the energy equality, and finally the uniqueness.

Proof of the existence and of the energy inequality. Let us consider the Galerkin approximate solutions

$$
\boldsymbol{w}_{m}(t, \boldsymbol{x})=\sum_{j=1}^{m} g_{m}^{j}(t) \boldsymbol{E}_{j}(\boldsymbol{x}), \quad \rho_{m}(t, \boldsymbol{x})=\sum_{j=1}^{m} \gamma_{m}^{j}(t) E_{j}(\boldsymbol{x}),
$$

where $\boldsymbol{E}_{j}$ and $E_{j}$ are smooth eigenfunctions of the Stokes and Laplace operator, respectively, in $D$, with Dirichlet boundary conditions on $\Gamma$ and periodicity in $x_{1}$ and $x_{2}$ (see Rummler [27]). If $\boldsymbol{P}_{m}$ denotes the projection on $\operatorname{span}\left\{\boldsymbol{E}_{1}, \ldots, \boldsymbol{E}_{m}\right\}$ and similarly for $P_{m}$, the couple $\left(\boldsymbol{w}_{m}, \rho_{m}\right)$ solves the Cauchy problem

$$
\begin{aligned}
\frac{\mathrm{d}}{\mathrm{d} t}\left(\boldsymbol{w}_{m}, \boldsymbol{E}_{i}\right)+\nu\left(\nabla \boldsymbol{w}_{m}, \nabla \boldsymbol{E}_{i}\right)-\left(\boldsymbol{w}_{m} \otimes \boldsymbol{w}_{m}, \nabla \overline{\boldsymbol{E}}_{i}^{\mathrm{h}}\right) & =-\left(\rho_{m} \boldsymbol{e}_{3}, \boldsymbol{E}_{i}\right), \\
\frac{\mathrm{d}}{\mathrm{d} t}\left(\rho_{m}, E_{i}\right)+K\left(\nabla \rho_{m}, \nabla E_{i}\right)-\left(\rho_{m} \boldsymbol{w}_{m}, \nabla \bar{E}_{i}^{\mathrm{h}}\right) & =0, \\
\boldsymbol{w}_{m}(0)=\boldsymbol{P}_{m}(\boldsymbol{w}(0)), \quad \rho_{m}(0) & =P_{m}(\rho(0)),
\end{aligned}
$$

for $i=1, \ldots, m$.

We test the first equation against $A_{\mathrm{h}} \boldsymbol{w}_{m}$ and use the following fundamental identity (see [1])

$$
\left.-\left(\boldsymbol{w}_{m} \otimes \boldsymbol{w}_{m}, \nabla{\overline{A_{\mathrm{h}} \boldsymbol{w}_{m}}}^{\mathrm{h}}\right)=\left(\overline{\nabla \cdot\left(\boldsymbol{w}_{m} \otimes \boldsymbol{w}_{m}\right.}\right)^{\mathrm{h}}, A_{\mathrm{h}} \boldsymbol{w}_{m}\right)=0,
$$

to get

$$
\begin{aligned}
& \frac{1}{2} \frac{\mathrm{d}}{\mathrm{d} t}\left(\left\|\boldsymbol{w}_{m}\right\|^{2}+\alpha^{2}\left\|\nabla_{\mathrm{h}} \boldsymbol{w}_{m}\right\|^{2}\right)+\nu\left(\left\|\nabla \boldsymbol{w}_{m}\right\|^{2}+\alpha^{2}\left\|\nabla_{\mathrm{h}} \nabla \boldsymbol{w}_{m}\right\|^{2}\right) \\
& =-\left(\rho_{m} \boldsymbol{e}_{3}, \boldsymbol{w}_{m}\right)-\alpha^{2}\left(\nabla_{\mathrm{h}} \rho_{m}, \nabla_{\mathrm{h}} \boldsymbol{w}_{m}\right) \\
& \leq \frac{1}{2}\left\|\rho_{m}\right\|^{2}+\frac{1}{2}\left\|\boldsymbol{w}_{m}\right\|^{2}+\frac{\alpha^{2}}{2}\left\|\nabla_{\mathrm{h}} \rho_{m}\right\|^{2}+\frac{\alpha^{2}}{2}\left\|\nabla_{\mathrm{h}} \boldsymbol{w}_{m}\right\|^{2} .
\end{aligned}
$$


We test the second equation against $A_{\mathrm{h}} \rho_{m}$ and use

$$
-\left(\overline{\rho_{m} \boldsymbol{w}_{m}}{ }^{\mathrm{h}}, A_{\mathrm{h}} \nabla \rho_{m}\right)=-\left(\rho_{m} \boldsymbol{w}_{m}, \nabla \rho_{m}\right)=0
$$

(due to $\nabla \cdot \boldsymbol{w}_{m}=0$; note that we need to test the equation for $\rho_{m}$ against $A_{\mathrm{h}} \rho_{m}$ in order to cancel the filter and obtain the previous identity) to get

$$
\frac{1}{2} \frac{\mathrm{d}}{\mathrm{d} t}\left(\left\|\rho_{m}\right\|^{2}+\alpha^{2}\left\|\nabla_{\mathrm{h}} \rho_{m}\right\|^{2}\right)+K\left(\left\|\nabla \rho_{m}\right\|^{2}+\alpha^{2}\left\|\nabla_{\mathrm{h}} \nabla \rho_{m}\right\|^{2}\right)=0 .
$$

Summing up the latest two inequalities, we obtain

$$
\begin{aligned}
& \frac{1}{2} \frac{\mathrm{d}}{\mathrm{d} t}\left(\left\|\boldsymbol{w}_{m}\right\|^{2}+\alpha^{2}\left\|\nabla_{\mathrm{h}} \boldsymbol{w}_{m}\right\|^{2}+\left\|\rho_{m}\right\|^{2}+\alpha^{2}\left\|\nabla_{\mathrm{h}} \rho_{m}\right\|^{2}\right) \\
& \quad+\nu\left(\left\|\nabla \boldsymbol{w}_{m}\right\|^{2}+\alpha^{2}\left\|\nabla_{\mathrm{h}} \nabla \boldsymbol{w}_{m}\right\|^{2}\right)+K\left(\left\|\nabla \rho_{m}\right\|^{2}+\alpha^{2}\left\|\nabla_{\mathrm{h}} \nabla \rho_{m}\right\|^{2}\right) \\
& \quad \leq \frac{1}{2}\left(\left\|\boldsymbol{w}_{m}\right\|^{2}+\alpha^{2}\left\|\nabla_{\mathrm{h}} \boldsymbol{w}_{m}\right\|^{2}+\left\|\rho_{m}\right\|^{2}+\alpha^{2}\left\|\nabla_{\mathrm{h}} \rho_{m}\right\|^{2}\right) .
\end{aligned}
$$

An application of the Gronwall lemma gives

$$
\begin{aligned}
& \left\|\boldsymbol{w}_{m}(t)\right\|^{2}+\alpha^{2}\left\|\nabla_{\mathrm{h}} \boldsymbol{w}_{m}(t)\right\|^{2}+\left\|\rho_{m}(t)\right\|^{2}+\alpha^{2}\left\|\nabla_{\mathrm{h}} \rho_{m}(t)\right\|^{2} \\
& \leq \mathrm{e}^{t}\left(\left\|\boldsymbol{w}_{m}(0)\right\|^{2}+\alpha^{2}\left\|\nabla_{\mathrm{h}} \boldsymbol{w}_{m}(0)\right\|^{2}+\left\|\rho_{m}(0)\right\|^{2}+\alpha^{2}\left\|\nabla_{\mathrm{h}} \rho_{m}(0)\right\|^{2}\right),
\end{aligned}
$$

so that $\boldsymbol{w}_{m} \in L^{\infty}\left(0, T ; V_{\mathrm{h}}\right)$ and $\rho_{m} \in L^{\infty}\left(0, T ; H_{\mathrm{h}}^{1}\right)$. Integrating (4) over $[0, T]$, we have $\boldsymbol{w}_{m} \in L^{2}\left(0, T ; V \cap\left(H_{\mathrm{h}}^{2}\right)^{3}\right)$ and $\rho_{m} \in L^{2}\left(0, T ; H^{1} \cap H_{\mathrm{h}}^{2}\right)$.

From $\nabla \cdot \boldsymbol{w}_{m}=0$, we get $\partial_{3} w_{m}^{3}=-\nabla_{\mathrm{h}} \cdot \boldsymbol{w}_{m}^{\mathrm{h}}$, hence $w_{m}^{3} \in L^{\infty}\left(0, T ; H^{1}\right)$, since $w_{m}^{3}=0$ on $\Gamma$, and $\partial_{3}^{2} w_{m}^{3}=-\nabla_{\mathrm{h}} \cdot \partial_{3} \boldsymbol{w}_{m}^{\mathrm{h}}$, thus $\Delta w_{m}^{3} \in L^{2}\left(0, T ; L^{2}\right)$, and finally $w_{m}^{3} \in L^{\infty}\left(0, T ; H^{1}\right) \cap L^{2}\left(0, T ; H^{2}\right)$.

Now we need estimates in order to pass to the limit as $m \rightarrow+\infty$. The nonlinear terms can be estimated using the Hölder and the Gagliardo-Nirenberg inequalities. We have (see [1])

$$
\begin{aligned}
& \left.\mid\left(\overline{\nabla \cdot\left(\boldsymbol{w}_{m} \otimes \boldsymbol{w}_{m}\right)}\right)^{\mathrm{h}}, \boldsymbol{\varphi}\right) \mid \\
& \leq C\left(\left\|\boldsymbol{w}_{m}^{\mathrm{h}}\right\|^{\frac{1}{2}}\left\|\nabla \boldsymbol{w}_{m}^{\mathrm{h}}\right\|^{\frac{1}{2}}\left\|\nabla_{\mathrm{h}} \boldsymbol{w}_{m}\right\|+\left\|w_{m}^{3}\right\|^{\frac{1}{2}}\left\|\nabla w_{m}^{3}\right\|^{\frac{1}{2}}\left\|\nabla \boldsymbol{w}_{m}\right\|\right)\|\nabla \boldsymbol{\varphi}\|,
\end{aligned}
$$

while $\int_{0}^{T}\left|\left(\rho_{m} \boldsymbol{e}_{3}, \boldsymbol{\varphi}\right)\right| \leq\left\|\rho_{m}\right\|_{L^{2} L^{2}}\|\boldsymbol{\varphi}\|_{L^{2} L^{2}}$, so we deduce that

$$
\partial_{t} \boldsymbol{w}_{m} \in L^{2}\left(0, T ; V^{*}\right) .
$$

Concerning $\partial_{t} \rho_{m}$, we have

$$
-\left({\overline{\rho_{m}}}^{\boldsymbol{w}_{m}} \mathrm{~h}, \nabla \psi\right)=\left(\nabla_{\mathrm{h}}{\overline{\left(\rho_{m} \boldsymbol{w}_{m}^{\mathrm{h}}\right.}}^{\mathrm{h}}, \psi\right)-\left({\overline{\rho_{m} w_{m}^{3}}}^{\mathrm{h}}, \partial_{3} \psi\right),
$$


and hence, by using the Hölder and the Gagliardo-Nirenberg inequalities,

$$
\begin{aligned}
& \left.\mid\left(\overline{\rho_{m}(t) \boldsymbol{w}_{m}(t)}\right)^{\mathrm{h}}, \nabla \psi(t)\right) \mid \\
& \leq C\left(\left\|\rho_{m}(t)\right\|_{L^{2}}\left\|\boldsymbol{w}_{m}^{\mathrm{h}}(t)\right\|_{L^{3}}\|\psi(t)\|_{L^{6}}+\left\|\rho_{m}(t)\right\|_{L^{2}}\left\|w_{m}^{3}(t)\right\|_{L^{\infty}}\|\nabla \psi(t)\|_{L^{2}}\right) \\
& \leq C\left\|\rho_{m}(t)\right\|_{L^{2}}\left(\left\|\boldsymbol{w}_{m}(t)\right\|_{L^{2}}^{\frac{1}{2}}\left\|\nabla \boldsymbol{w}_{m}(t)\right\|_{L^{2}}^{\frac{1}{2}}\|\nabla \psi(t)\|_{L^{2}}\right. \\
& \left.\quad+\left\|\Delta w_{m}^{3}(t)\right\|_{L^{2}}^{\frac{3}{4}}\left\|w_{m}^{3}(t)\right\|_{L^{2}}^{\frac{1}{4}}\|\nabla \psi(t)\|_{L^{2}}\right) \\
& \leq C\left\|\rho_{m}(t)\right\|_{L^{2}}\left(\left\|\boldsymbol{w}_{m}(t)\right\|_{V}+\left\|w_{m}^{3}(t)\right\|_{H^{2}}\right)\|\nabla \psi(t)\|_{L^{2}},
\end{aligned}
$$

so that

$$
\begin{aligned}
& \int_{0}^{T}\left|\left(\overline{\rho_{m}(t) \boldsymbol{w}_{m}(t)}, \nabla \psi(t)\right)\right| \mathrm{d} t \\
& \leq C\left\|\rho_{m}\right\|_{L^{\infty} L^{2}}\left(\left\|\boldsymbol{w}_{m}\right\|_{L^{2} V}+\left\|w_{m}^{3}(t)\right\|_{L^{2} H^{2}}\right)\|\nabla \psi(t)\|_{L^{2} L^{2}}
\end{aligned}
$$

Finally, to estimate $\left|\left(\nabla \rho_{m}, \nabla \psi\right)\right|$, we use $\rho_{m} \in L^{2}\left(0, T ; H^{1}\right)$ to get

$$
\int_{0}^{T}\left|\left(\nabla \rho_{m}(t), \nabla \psi(t)\right)\right| \mathrm{d} t \leq\left\|\nabla \rho_{m}\right\|_{L^{2} L^{2}}\|\nabla \psi\|_{L^{2} L^{2}}
$$

so that $\partial_{t} \rho_{m} \in L^{2}\left(0, T ; H^{-1}\right)$.

The estimates for the time derivatives and standard compactness results (as the Aubin-Lions lemma, see [16]; and it is here that we need $\nu, K>0$ in order to have the required compact embeddings) imply that there exist $\boldsymbol{w}$ and $\rho$ regular as in the definition of regular weak solutions so that, up to sub-sequences,

$$
\boldsymbol{w}_{m} \rightarrow \boldsymbol{w}, \quad \rho_{m} \rightarrow \rho, \quad \text { in } L^{2}\left(0, T ; L^{2}(D)\right), \text { as } m \rightarrow+\infty .
$$

This is sufficient to pass to the limit in the nonlinear terms and get that $(\boldsymbol{w}, \rho)$ is a regular weak solution to the model. Moreover, by using standard lower semicontinuity results for norms, we obtain that such a solution satisfies the energy inequality

$$
\begin{aligned}
& \frac{1}{2}\left(\|\boldsymbol{w}(t)\|^{2}+\alpha^{2}\left\|\nabla_{\mathrm{h}} \boldsymbol{w}(t)\right\|^{2}+\|\rho(t)\|^{2}+\alpha^{2}\left\|\nabla_{\mathrm{h}} \rho(t)\right\|^{2}\right) \\
& \quad+\nu \int_{0}^{t}\left(\|\nabla \boldsymbol{w}(s)\|^{2}+\alpha^{2}\left\|\nabla_{\mathrm{h}} \nabla \boldsymbol{w}(s)\right\|^{2}\right) \mathrm{d} s+K \int_{0}^{t}\left(\|\nabla \rho(s)\|^{2}+\alpha^{2}\left\|\nabla_{\mathrm{h}} \nabla \rho(s)\right\|^{2}\right) \mathrm{d} s \\
& \leq \frac{1}{2}\left(\|\boldsymbol{w}(0)\|^{2}+\alpha^{2}\left\|\nabla_{\mathrm{h}} \boldsymbol{w}(0)\right\|^{2}+\|\rho(0)\|^{2}+\alpha^{2}\left\|\nabla_{\mathrm{h}} \rho(0)\right\|^{2}\right) \\
& \quad-\int_{0}^{t}\left(A_{\mathrm{h}}^{\frac{1}{2}} \rho(s) \boldsymbol{e}_{3}, A_{\mathrm{h}}^{\frac{1}{2}} \boldsymbol{w}(s)\right) \mathrm{d} s .
\end{aligned}
$$

Finally, we can easily deduce $\boldsymbol{w} \in C_{\mathrm{w}}\left(0, T ; V_{\mathrm{h}}\right)$ and also that $\rho \in C_{\mathrm{w}}\left(0, T ; H_{\mathrm{h}}^{1}\right)$. 
On the energy identity. We now show that in addition to the energy inequality, we have for this model an exact balance of the energy, hence the energy of the model identity. Let $\boldsymbol{w}_{\varepsilon}, \rho_{\varepsilon}$ denote the standard regularization (convolution in time) of $\boldsymbol{w}, \rho$ (and so on), with $0<t_{0}<t_{1}<T$ fixed, and $0<\varepsilon<t_{0}$, $\varepsilon<T-t_{1}, \varepsilon<t_{1}-t_{0}$ (see [1]). For each $t \in\left[t_{0}, t_{1}\right]$, we have

$$
\boldsymbol{w}_{\varepsilon}(t)=\left(j_{\varepsilon} * \boldsymbol{w}\right)(t)=\int_{t_{0}}^{t_{1}} j_{\varepsilon}(t-s) \boldsymbol{w}(s) \mathrm{d} s,
$$

where the smooth function $j_{\varepsilon}$ is even, positive, supported in $]-\varepsilon, \varepsilon[$, and such that $\int_{-\varepsilon}^{\varepsilon} j_{\varepsilon}(s) \mathrm{d} s=1$. Under these assumptions, for any $\boldsymbol{w} \in L^{q}\left(t_{0}, t_{1} ; X\right)$, with $1 \leq q<+\infty$ and $X$ Hilbert space, we have the following properties (see [20]):

- $\boldsymbol{w}_{\varepsilon} \in C^{\infty}\left(\left[t_{0}, t_{1}\right] ; X\right)$;

- $\lim _{\varepsilon \rightarrow 0}\left\|\boldsymbol{w}_{\varepsilon}-\boldsymbol{w}\right\|_{L^{q}\left(t_{0}, t_{1} ; X\right)}=0$;

- $\lim _{k \rightarrow+\infty}\left\|\left[\boldsymbol{w}_{k}\right]_{\varepsilon}-\boldsymbol{w}_{\varepsilon}\right\|_{L^{q}\left(t_{0}, t_{1} ; X\right)}=0$ for each sub-sequence $\boldsymbol{w}_{k} \in L^{q}\left(t_{0}, t_{1} ; X\right)$ such that $\boldsymbol{w}_{k} \rightarrow \boldsymbol{w}$ in $L^{q}\left(t_{0}, t_{1} ; X\right)$.

Let be given

$$
\begin{array}{cl}
\boldsymbol{w}_{k} \rightarrow \boldsymbol{w} & \text { in } L^{\infty}\left(0, T ; V_{\mathrm{h}}\right) \cap L^{2}\left(0, T ; V \cap\left(H_{\mathrm{h}}^{2}\right)^{3}\right), \\
\rho_{k} \rightarrow \rho & \text { in } L^{\infty}\left(0, T ; H_{\mathrm{h}}^{1}\right) \cap L^{2}\left(0, T ; H^{1} \cap H_{\mathrm{h}}^{2}\right),
\end{array}
$$

and test the weak formulation against $A_{\mathrm{h}} \boldsymbol{w}_{k, \varepsilon}$ in the first equation and $A_{\mathrm{h}} \rho_{k, \varepsilon}$ in the second one:

$$
\begin{aligned}
& \int_{t_{0}}^{t_{1}}\left\{\left(\boldsymbol{w}, \partial_{t} A_{\mathrm{h}} \boldsymbol{w}_{k, \varepsilon}\right)-\nu\left(\nabla \boldsymbol{w}, \nabla A_{\mathrm{h}} \boldsymbol{w}_{k, \varepsilon}\right)+\left(\boldsymbol{w} \otimes \boldsymbol{w}, \nabla \boldsymbol{w}_{k, \varepsilon}\right)\right\}(s) \mathrm{d} s \\
& =\int_{t_{0}}^{t_{1}}\left(\rho \boldsymbol{e}_{3}, A_{\mathrm{h}} \boldsymbol{w}_{k, \varepsilon}\right)(s) \mathrm{d} s+\left(\boldsymbol{w}\left(t_{1}\right), A_{\mathrm{h}} \boldsymbol{w}_{k, \varepsilon}\left(t_{1}\right)\right)-\left(\boldsymbol{w}\left(t_{0}\right), A_{\mathrm{h}} \boldsymbol{w}_{k, \varepsilon}\left(t_{0}\right)\right), \\
& \int_{t_{0}}^{t_{1}}\left\{\left(\rho, \partial_{t} A_{\mathrm{h}} \rho_{k, \varepsilon}\right)+\left(\rho \boldsymbol{w}, \nabla \rho_{k, \varepsilon}\right)-K\left(\nabla \rho, \nabla A_{\mathrm{h}} \rho_{k, \varepsilon}\right)\right\}(s) \mathrm{d} s \\
& =\left(\rho\left(t_{1}\right), A_{\mathrm{h}} \rho_{k, \varepsilon}\left(t_{1}\right)\right)-\left(\rho\left(t_{0}\right), A_{\mathrm{h}} \rho_{k, \varepsilon}\left(t_{0}\right)\right) .
\end{aligned}
$$

Summing up and using $A_{\mathrm{h}}=I-\alpha^{2} \Delta_{\mathrm{h}}$ yields

$$
\begin{aligned}
& \int_{t_{0}}^{t_{1}}\left\{\left(\boldsymbol{w}, \partial_{t} \boldsymbol{w}_{k, \varepsilon}\right)+\alpha^{2}\left(\nabla_{\mathrm{h}} \boldsymbol{w}, \partial_{t} \nabla_{\mathrm{h}} \boldsymbol{w}_{k, \varepsilon}\right)-\nu\left(\nabla \boldsymbol{w}, \nabla \boldsymbol{w}_{k, \varepsilon}\right)\right. \\
& -\nu \alpha^{2}\left(\nabla_{\mathrm{h}} \nabla \boldsymbol{w}, \nabla_{\mathrm{h}} \nabla \boldsymbol{w}_{k, \varepsilon}\right)+\left((\boldsymbol{w} \cdot \nabla) \boldsymbol{w}_{k, \varepsilon}, \boldsymbol{w}\right)+\left(\rho, \partial_{t} \rho_{k, \varepsilon}\right)+\alpha^{2}\left(\nabla_{\mathrm{h}} \rho, \partial_{t} \nabla_{\mathrm{h}} \rho_{k, \varepsilon}\right) \\
& \left.\quad+\left(\rho \boldsymbol{w}, \nabla \rho_{k, \varepsilon}\right)-K\left(\nabla \rho, \nabla \rho_{k, \varepsilon}\right)-K \alpha^{2}\left(\nabla_{\mathrm{h}} \nabla \rho, \nabla_{\mathrm{h}} \nabla \rho_{k, \varepsilon}\right)\right\}(s) \mathrm{d} s \\
& =\left(\boldsymbol{w}\left(t_{1}\right), \boldsymbol{w}_{k, \varepsilon}\left(t_{1}\right)\right)+\alpha^{2}\left(\nabla_{\mathrm{h}} \boldsymbol{w}\left(t_{1}\right), \nabla_{\mathrm{h}} \boldsymbol{w}_{k, \varepsilon}\left(t_{1}\right)\right)+\left(\rho\left(t_{1}\right), \rho_{k, \varepsilon}\left(t_{1}\right)\right) \\
& \quad+\alpha^{2}\left(\nabla_{\mathrm{h}} \rho\left(t_{1}\right), \nabla_{\mathrm{h}} \rho_{k, \varepsilon}\left(t_{1}\right)\right)-\left(\boldsymbol{w}\left(t_{0}\right), \boldsymbol{w}_{k, \varepsilon}\left(t_{0}\right)\right)-\alpha^{2}\left(\nabla_{\mathrm{h}} \boldsymbol{w}\left(t_{0}\right), \nabla_{\mathrm{h}} \boldsymbol{w}_{k, \varepsilon}\left(t_{0}\right)\right) \\
& \quad-\left(\rho\left(t_{0}\right), \rho_{k, \varepsilon}\left(t_{0}\right)\right)-\alpha^{2}\left(\nabla_{\mathrm{h}} \rho\left(t_{0}\right), \nabla_{\mathrm{h}} \rho_{k, \varepsilon}\left(t_{0}\right)\right)+\int_{t_{0}}^{t_{1}}\left(\rho \boldsymbol{e}_{3}, A_{\mathrm{h}} \boldsymbol{w}_{k, \varepsilon}\right)(s) \mathrm{d} s .
\end{aligned}
$$


Remark 2.3. When $K=0$, the term $\left(\rho \boldsymbol{w}, \nabla \rho_{k, \varepsilon}\right)$ is problematic, since we need the convergence property $\rho_{k} \rightarrow \rho$ in $L^{2}\left(0, T ; H^{1} \cap H_{\mathrm{h}}^{2}\right)$, while we have only convergence in $L^{2}\left(0, T ; H_{\mathrm{h}}^{1}\right)$, for $K=0$. This is an additional reason to assume $K>0$.

We show now that

$$
\lim _{k \rightarrow+\infty} \int_{t_{0}}^{t_{1}}\left(\rho \boldsymbol{w}, \nabla \rho_{k, \varepsilon}\right)(s) \mathrm{d} s=\int_{t_{0}}^{t_{1}}\left(\rho \boldsymbol{w}, \nabla \rho_{\varepsilon}\right)(s) \mathrm{d} s .
$$

In fact, for each $s \in\left[t_{0}, t_{1}\right]$, we have

$$
\begin{aligned}
& \left|\left(\rho \boldsymbol{w}, \nabla\left(\rho_{k, \varepsilon}-\rho_{\varepsilon}\right)\right)\right| \leq\left|\left(\rho \boldsymbol{w}^{\mathrm{h}}, \nabla_{\mathrm{h}}\left(\rho_{k, \varepsilon}-\rho_{\varepsilon}\right)\right)\right|+\left|\left(\rho w^{3}, \partial_{3}\left(\rho_{k, \varepsilon}-\rho_{\varepsilon}\right)\right)\right| \\
& \leq\|\rho\|_{L^{2}}\|\boldsymbol{w}\|_{L^{3}}\left\|\nabla_{\mathrm{h}}\left(\rho_{k, \varepsilon}-\rho_{\varepsilon}\right)\right\|_{L^{6}}+\|\rho\|_{L^{3}}\left\|w^{3}\right\|_{L^{6}}\left\|\partial_{3}\left(\rho_{k, \varepsilon}-\rho_{\varepsilon}\right)\right\|_{L^{2}} \\
& \leq C\left\{\|\rho\|_{L^{2}}\|\boldsymbol{w}\|_{L^{2}}^{\frac{1}{2}}\|\nabla \boldsymbol{w}\|_{L^{2}}^{\frac{1}{2}}\left\|\nabla_{\mathrm{h}} \nabla\left(\rho_{k, \varepsilon}-\rho_{\varepsilon}\right)\right\|_{L^{2}}\right. \\
& \left.\quad+\|\rho\|_{L^{2}}^{\frac{1}{2}}\|\nabla \rho\|_{L^{2}}^{\frac{1}{2}}\left\|\nabla w^{3}\right\|_{L^{2}}\left\|\nabla\left(\rho_{k, \varepsilon}-\rho_{\varepsilon}\right)\right\|_{L^{2}}\right\} \\
& \leq C\left\{\|\rho\|_{L^{2}}\|\boldsymbol{w}\|_{V}\left\|\rho_{k, \varepsilon}-\rho_{\varepsilon}\right\|_{H_{\mathrm{h}}^{2}}+\|\rho\|_{H^{1}}\left\|w^{3}\right\|_{H^{1}}\left\|\rho_{k, \varepsilon}-\rho_{\varepsilon}\right\|_{H^{1}}\right\} .
\end{aligned}
$$

Hence

$$
\begin{aligned}
& \int_{t_{0}}^{t_{1}}\left|\left(\rho \boldsymbol{w}, \nabla\left(\rho_{k, \varepsilon}-\rho_{\varepsilon}\right)\right)(s)\right| \mathrm{d} s \\
& \leq C\left\{\|\rho\|_{L^{\infty} L^{2}}\|\boldsymbol{w}\|_{L^{2} V}\left\|\rho_{k, \varepsilon}-\rho_{\varepsilon}\right\|_{L^{2} H_{\mathrm{h}}^{2}}+\|\rho\|_{L^{2} H^{1}}\left\|w^{3}\right\|_{L^{\infty} H^{1}}\left\|\rho_{k, \varepsilon}-\rho_{\varepsilon}\right\|_{L^{2} H^{1}}\right\},
\end{aligned}
$$

and this implies (5).

Moreover, since

$$
\lim _{k \rightarrow+\infty} \int_{t_{0}}^{t_{1}}\left((\boldsymbol{w} \cdot \nabla) \boldsymbol{w}_{k, \varepsilon}, \boldsymbol{w}\right)(s) \mathrm{d} s=\int_{t_{0}}^{t_{1}}\left((\boldsymbol{w} \cdot \nabla) \boldsymbol{w}_{\varepsilon}, \boldsymbol{w}\right)(s) \mathrm{d} s,
$$

as in [1], and by observing that the other terms do not give any problem (since they are linear terms and we have convergence in appropriate spaces), by taking the limit as $k \rightarrow+\infty$, we get

$$
\begin{aligned}
\int_{t_{0}}^{t_{1}} & \left\{\left(\boldsymbol{w}, \partial_{t} \boldsymbol{w}_{\varepsilon}\right)+\alpha^{2}\left(\nabla_{\mathrm{h}} \boldsymbol{w}, \partial_{t} \nabla_{\mathrm{h}} \boldsymbol{w}_{\varepsilon}\right)-\nu\left(\nabla \boldsymbol{w}, \nabla \boldsymbol{w}_{\varepsilon}\right)-\nu \alpha^{2}\left(\nabla_{\mathrm{h}} \nabla \boldsymbol{w}, \nabla_{\mathrm{h}} \nabla \boldsymbol{w}_{\varepsilon}\right)\right. \\
& +\left((\boldsymbol{w} \cdot \nabla) \boldsymbol{w}_{\varepsilon}, \boldsymbol{w}\right)+\left(\rho, \partial_{t} \rho_{\varepsilon}\right)+\alpha^{2}\left(\nabla_{\mathrm{h}} \rho, \partial_{t} \nabla_{\mathrm{h}} \rho_{\varepsilon}\right)+\left(\rho \boldsymbol{w}, \nabla \rho_{\varepsilon}\right) \\
& \left.-K\left(\nabla \rho, \nabla \rho_{\varepsilon}\right)-K \alpha^{2}\left(\nabla_{\mathrm{h}} \nabla \rho, \nabla_{\mathrm{h}} \nabla \rho_{\varepsilon}\right)\right\}(s) \mathrm{d} s \\
= & \left(\boldsymbol{w}\left(t_{1}\right), \boldsymbol{w}_{\varepsilon}\left(t_{1}\right)\right)+\alpha^{2}\left(\nabla_{\mathrm{h}} \boldsymbol{w}\left(t_{1}\right), \nabla_{\mathrm{h}} \boldsymbol{w}_{\varepsilon}\left(t_{1}\right)\right)+\left(\rho\left(t_{1}\right), \rho_{\varepsilon}\left(t_{1}\right)\right) \\
& +\alpha^{2}\left(\nabla_{\mathrm{h}} \rho\left(t_{1}\right), \nabla_{\mathrm{h}} \rho_{\varepsilon}\left(t_{1}\right)\right)-\left(\boldsymbol{w}\left(t_{0}\right), \boldsymbol{w}_{\varepsilon}\left(t_{0}\right)\right)-\alpha^{2}\left(\nabla_{\mathrm{h}} \boldsymbol{w}\left(t_{0}\right), \nabla_{\mathrm{h}} \boldsymbol{w}_{\varepsilon}\left(t_{0}\right)\right) \\
& -\left(\rho\left(t_{0}\right), \rho_{\varepsilon}\left(t_{0}\right)\right)-\alpha^{2}\left(\nabla_{\mathrm{h}} \rho\left(t_{0}\right), \nabla_{\mathrm{h}} \rho_{\varepsilon}\left(t_{0}\right)\right)+\int_{t_{0}}^{t_{1}}\left(A_{\mathrm{h}}^{\frac{1}{2}} \rho \boldsymbol{e}_{3}, A_{\mathrm{h}}^{\frac{1}{2}} \boldsymbol{w}_{\varepsilon}\right)(s) \mathrm{d} s
\end{aligned}
$$


We are going to show that

$$
\begin{gathered}
\int_{t_{0}}^{t_{1}}\left\{\left(\boldsymbol{w}, \partial_{t} \boldsymbol{w}_{\varepsilon}\right)+\alpha^{2}\left(\nabla_{\mathrm{h}} \boldsymbol{w}, \partial_{t} \nabla_{\mathrm{h}} \boldsymbol{w}_{\varepsilon}\right)+\left(\rho, \partial_{t} \rho_{\varepsilon}\right)+\alpha^{2}\left(\nabla_{\mathrm{h}} \rho, \partial_{t} \nabla_{\mathrm{h}} \rho_{\varepsilon}\right)\right\}(s) \mathrm{d} s=0, \\
\lim _{\varepsilon \rightarrow 0} \int_{t_{0}}^{t_{1}}\left\{\left((\boldsymbol{w} \cdot \nabla) \boldsymbol{w}_{\varepsilon}, \boldsymbol{w}\right)+\left(\rho \boldsymbol{w}, \nabla \rho_{\varepsilon}\right)\right\}(s) \mathrm{d} s=0 \\
\lim _{\varepsilon \rightarrow 0} \int_{t_{0}}^{t_{1}}\left\{A_{\mathrm{h}}^{\frac{1}{2}} \rho \boldsymbol{e}_{3}, A_{\mathrm{h}}^{\frac{1}{2}} \boldsymbol{w}_{\varepsilon}\right)(s) \mathrm{d} s=\int_{t_{0}}^{t_{1}}\left(A_{\mathrm{h}}^{\frac{1}{2}} \rho(s) \boldsymbol{e}_{3}, A_{\mathrm{h}}^{\frac{1}{2}} \boldsymbol{w}(s)\right) \mathrm{d} s \\
\lim _{\varepsilon \rightarrow 0} \int_{t_{0}}^{t_{1}}\left\{-\nu\left(\nabla \boldsymbol{w}, \nabla \boldsymbol{w}_{\varepsilon}\right)-\nu \alpha^{2}\left(\nabla_{\mathrm{h}} \nabla \boldsymbol{w}, \nabla_{\mathrm{h}} \nabla \boldsymbol{w}_{\varepsilon}\right)\right. \\
\left.-K\left(\nabla \rho, \nabla \rho_{\varepsilon}\right)-K \alpha^{2}\left(\nabla_{\mathrm{h}} \nabla \rho, \nabla_{\mathrm{h}} \nabla \rho_{\varepsilon}\right)\right\}(s) \mathrm{d} s \\
=-\nu \int_{t_{0}}^{t_{1}}\left\{\|\nabla \boldsymbol{w}(s)\|^{2}+\alpha^{2}\left\|\nabla_{\mathrm{h}} \nabla \boldsymbol{w}(s)\right\|^{2}\right\} \mathrm{d} s \\
-K \int_{t_{0}}^{t_{1}}\left\{\|\nabla \rho(s)\|^{2}+\alpha^{2}\left\|\nabla_{\mathrm{h}} \nabla \rho(s)\right\|^{2}\right\} \mathrm{d} s
\end{gathered}
$$

and

$$
\begin{aligned}
& \left(\boldsymbol{w}\left(t_{1}\right), \boldsymbol{w}_{\varepsilon}\left(t_{1}\right)\right)+\alpha^{2}\left(\nabla_{\mathrm{h}} \boldsymbol{w}\left(t_{1}\right), \nabla_{\mathrm{h}} \boldsymbol{w}_{\varepsilon}\left(t_{1}\right)\right) \\
& \quad+\left(\rho\left(t_{1}\right), \rho_{\varepsilon}\left(t_{1}\right)\right)+\alpha^{2}\left(\nabla_{\mathrm{h}} \rho\left(t_{1}\right), \nabla_{\mathrm{h}} \rho_{\varepsilon}\left(t_{1}\right)\right) \\
& =\frac{1}{2}\left\{\left\|\boldsymbol{w}\left(t_{1}\right)\right\|^{2}+\alpha^{2}\left\|\nabla_{\mathrm{h}} \boldsymbol{w}\left(t_{1}\right)\right\|^{2}+\left\|\rho\left(t_{1}\right)\right\|^{2}+\alpha^{2}\left\|\nabla_{\mathrm{h}} \rho\left(t_{1}\right)\right\|^{2}\right\}+O(\varepsilon), \\
& \left(\boldsymbol{w}\left(t_{0}\right), \boldsymbol{w}_{\varepsilon}\left(t_{0}\right)\right)+\alpha^{2}\left(\nabla_{\mathrm{h}} \boldsymbol{w}\left(t_{0}\right), \nabla_{\mathrm{h}} \boldsymbol{w}_{\varepsilon}\left(t_{0}\right)\right) \\
& \quad+\left(\rho\left(t_{0}\right), \rho_{\varepsilon}\left(t_{0}\right)\right)+\alpha^{2}\left(\nabla_{\mathrm{h}} \rho\left(t_{0}\right), \nabla_{\mathrm{h}} \rho_{\varepsilon}\left(t_{0}\right)\right) \\
& =\frac{1}{2}\left\{\left\|\boldsymbol{w}\left(t_{0}\right)\right\|^{2}+\alpha^{2}\left\|\nabla_{\mathrm{h}} \boldsymbol{w}\left(t_{0}\right)\right\|^{2}+\left\|\rho\left(t_{0}\right)\right\|^{2}+\alpha^{2}\left\|\nabla_{\mathrm{h}} \rho\left(t_{0}\right)\right\|^{2}\right\}+O(\varepsilon),
\end{aligned}
$$

so that, substituting in (6) and taking the limit $\varepsilon \rightarrow 0$, we get the energy identity for the model:

$$
\begin{aligned}
& \frac{1}{2}\left\{\left\|\boldsymbol{w}\left(t_{1}\right)\right\|^{2}+\alpha^{2}\left\|\nabla_{\mathrm{h}} \boldsymbol{w}\left(t_{1}\right)\right\|^{2}+\left\|\rho\left(t_{1}\right)\right\|^{2}+\alpha^{2}\left\|\nabla_{\mathrm{h}} \rho\left(t_{1}\right)\right\|^{2}\right\} \\
& \quad+\int_{t_{0}}^{t_{1}}\left\{\nu\|\nabla \boldsymbol{w}(s)\|^{2}+\nu \alpha^{2}\left\|\nabla_{\mathrm{h}} \nabla \boldsymbol{w}(s)\right\|^{2}+K\|\nabla \rho(s)\|^{2}+K \alpha^{2}\left\|\nabla_{\mathrm{h}} \nabla \rho(s)\right\|^{2}\right\} \mathrm{d} s \\
& =\frac{1}{2}\left\{\left\|\boldsymbol{w}\left(t_{0}\right)\right\|^{2}+\alpha^{2}\left\|\nabla_{\mathrm{h}} \boldsymbol{w}\left(t_{0}\right)\right\|^{2}+\left\|\rho\left(t_{0}\right)\right\|^{2}+\alpha^{2}\left\|\nabla_{\mathrm{h}} \rho\left(t_{0}\right)\right\|^{2}\right\} \\
& \quad-\int_{t_{0}}^{t_{1}}\left(A_{\mathrm{h}}^{\frac{1}{2}} \rho(s) \boldsymbol{e}_{3}, A_{\mathrm{h}}^{\frac{1}{2}} \boldsymbol{w}(s)\right) \mathrm{d} s .
\end{aligned}
$$


Taking $t_{0} \rightarrow 0$ and $t_{1}=t$, thanks to the weak continuity of $\boldsymbol{w}, \rho$, we conclude

$$
\begin{aligned}
& \frac{1}{2}\left\{\|\boldsymbol{w}(t)\|^{2}+\alpha^{2}\left\|\nabla_{\mathrm{h}} \boldsymbol{w}(t)\right\|^{2}+\|\rho(t)\|^{2}+\alpha^{2}\left\|\nabla_{\mathrm{h}} \rho(t)\right\|^{2}\right\} \\
& \quad+\int_{0}^{t}\left\{\nu\|\nabla \boldsymbol{w}(s)\|^{2}+\nu \alpha^{2}\left\|\nabla_{\mathrm{h}} \nabla \boldsymbol{w}(s)\right\|^{2}+K\|\nabla \rho(s)\|^{2}+K \alpha^{2}\left\|\nabla_{\mathrm{h}} \nabla \rho(s)\right\|^{2}\right\} \mathrm{d} s \\
& =\frac{1}{2}\left\{\|\boldsymbol{w}(0)\|^{2}+\alpha^{2}\left\|\nabla_{\mathrm{h}} \boldsymbol{w}(0)\right\|^{2}+\|\rho(0)\|^{2}+\alpha^{2}\left\|\nabla_{\mathrm{h}} \rho(0)\right\|^{2}\right\} \\
& \quad-\int_{0}^{t}\left(A_{\mathrm{h}}^{\frac{1}{2}} \rho(s) \boldsymbol{e}_{3}, A_{\mathrm{h}}^{\frac{1}{2}} \boldsymbol{w}(s)\right) \mathrm{d} s .
\end{aligned}
$$

Now we prove the previous relations. Equations (10) and (9) follow immediately because of the convergence properties of $\boldsymbol{w}_{\varepsilon}$ and $\rho_{\varepsilon}$. By using just the regularity of $\boldsymbol{w}$ and $\rho$, we have the convergence

$$
\begin{aligned}
& \lim _{\varepsilon \rightarrow 0} \int_{t_{0}}^{t_{1}}\left\{\left((\boldsymbol{w} \cdot \nabla) \boldsymbol{w}_{\varepsilon}, \boldsymbol{w}\right)+\left(\rho \boldsymbol{w}, \nabla \rho_{\varepsilon}\right)\right\}(s) \mathrm{d} s \\
& =\int_{t_{0}}^{t_{1}}\{((\boldsymbol{w} \cdot \nabla) \boldsymbol{w}, \boldsymbol{w})+(\rho \boldsymbol{w}, \nabla \rho)\}(s) \mathrm{d} s \\
& =0
\end{aligned}
$$

where the equality to zero is obtained in a standard way by approximating $\boldsymbol{w}, \rho$ through smooth functions and using the fact that $\nabla \cdot \boldsymbol{w}=0$; thus we have (8). As for (7), let us observe that $j_{\varepsilon}$ is supported in $]-\varepsilon, \varepsilon[$ and even, so that its derivative $j_{\varepsilon}^{\prime}$ is odd. Recalling the definition of $\boldsymbol{w}_{\varepsilon}$, we deduce

$$
\begin{aligned}
\int_{t_{0}}^{t_{1}}\left(\boldsymbol{w}(s), \partial_{t} \boldsymbol{w}_{\varepsilon}(s)\right) \mathrm{d} s & =\int_{t_{0}}^{t_{1}} \int_{t_{0}}^{t_{1}} j_{\varepsilon}^{\prime}(s-r)(\boldsymbol{w}(s), \boldsymbol{w}(r)) \mathrm{d} s \mathrm{~d} r \\
& =\iint_{E_{1}}+\iint_{E_{2}} j_{\varepsilon}^{\prime}(s-r)(\boldsymbol{w}(s), \boldsymbol{w}(r)) \mathrm{d} s \mathrm{~d} r \\
& =0
\end{aligned}
$$

where

$$
\begin{aligned}
& E_{1}=\left\{(r, s) \in\left[t_{0}, t_{1}\right] \times\left[t_{0}, t_{1}\right]: r \leq s \leq r+\varepsilon\right\}, \\
& E_{2}=\left\{(r, s) \in\left[t_{0}, t_{1}\right] \times\left[t_{0}, t_{1}\right]: r-\varepsilon \leq s \leq r, r \leq t_{1}\right\} .
\end{aligned}
$$

Indeed, note that $E_{1}$ is symmetric to $E_{2}$ with respect to $s=r$, and $j_{\varepsilon}^{\prime}(s-r)$ is odd with respect to $s-r$, hence $\iint_{E_{2}} \cdots=-\iint_{E_{1}} \cdots$. Similarly, we deal with the remaining terms in (7).

Finally, we prove that $\left(\boldsymbol{w}\left(t_{1}\right), \boldsymbol{w}_{\varepsilon}\left(t_{1}\right)\right)=\frac{1}{2}\left\|\boldsymbol{w}\left(t_{1}\right)\right\|^{2}+O(\varepsilon)$; the other terms in (11) and (12) can be handled in the same way. First, using the fact that $j_{\varepsilon}$ is even and then performing the parameter change $r=s-t_{1}$, we have that 
$\boldsymbol{w}_{\varepsilon}\left(t_{1}\right)=\int_{t_{0}}^{t_{1}} j_{\varepsilon}\left(t_{1}-s\right) \boldsymbol{w}(s) \mathrm{d} s=\int_{t_{0}-t_{1}}^{0} j_{\varepsilon}(r) \boldsymbol{w}\left(r+t_{1}\right) \mathrm{d} r=\int_{-\varepsilon}^{0} j_{\varepsilon}(r) \boldsymbol{w}\left(r+t_{1}\right) \mathrm{d} r$, since $j_{\varepsilon}$ is supported in $]-\varepsilon, \varepsilon\left[\right.$ and $t_{0}-t_{1}<-\varepsilon$. Thus

$$
\begin{aligned}
\left(\boldsymbol{w}\left(t_{1}\right), \boldsymbol{w}_{\varepsilon}\left(t_{1}\right)\right) & =\int_{-\varepsilon}^{0} j_{\varepsilon}(r)\left(\boldsymbol{w}\left(t_{1}\right), \boldsymbol{w}\left(r+t_{1}\right)\right) \mathrm{d} r \\
& =\int_{-\varepsilon}^{0} j_{\varepsilon}(r)\left(\boldsymbol{w}\left(t_{1}\right), \boldsymbol{w}\left(t_{1}\right)\right) \mathrm{d} r+\int_{-\varepsilon}^{0} j_{\varepsilon}(r)\left(\boldsymbol{w}\left(t_{1}\right), \boldsymbol{w}\left(r+t_{1}\right)-\boldsymbol{w}\left(t_{1}\right)\right) \mathrm{d} r \\
& =\frac{1}{2}\left\|\boldsymbol{w}\left(t_{1}\right)\right\|^{2}+O(\varepsilon),
\end{aligned}
$$

where we have used $\int_{-\varepsilon}^{0} j_{\varepsilon}(r) \mathrm{d} r=\frac{1}{2}$ for the first term and $\boldsymbol{w} \in C_{\mathrm{w}}\left(0, T ; V_{\mathrm{h}}\right)$ for the second one.

Uniqueness and continuous dependence when $\mathbf{K}>\mathbf{0}$. Let us consider two solutions $\left(\boldsymbol{w}_{1}, \rho_{1}\right)$ and $\left(\boldsymbol{w}_{2}, \rho_{2}\right)$ to $(1),(2),(3)$ with the same initial data $\left(\boldsymbol{w}_{1}(0), \rho_{1}(0)\right)=\left(\boldsymbol{w}_{2}(0), \rho_{2}(0)\right)$, and set $\boldsymbol{w}:=\boldsymbol{w}_{1}-\boldsymbol{w}_{2}$ and $\rho:=\rho_{1}-\rho_{2}$, so that $(\boldsymbol{w}(0), \rho(0))=(\mathbf{0}, 0)$. We test the equations for $\partial_{t} \boldsymbol{w}_{1}$ and $\partial_{t} \boldsymbol{w}_{2}$ against $\left[A_{\mathrm{h}} \boldsymbol{w}\right]_{k, \varepsilon}$ and the equations for $\partial_{t} \rho_{1}$ and $\partial_{t} \rho_{2}$ against $\left[A_{\mathrm{h}} \rho\right]_{k, \varepsilon}$ (note that $A_{\mathrm{h}} \boldsymbol{w}$ and $A_{\mathrm{h}} \rho$ are not directly allowed as test functions). Observe that the $\varepsilon$ regularization commutes with the filter $A_{\mathrm{h}}$ and more generally with space derivatives. Using the same arguments as in the previous subsection, we can pass to the limit $k \rightarrow+\infty$. The equation for $\partial_{t} \boldsymbol{w}_{1}$ becomes

$$
\begin{aligned}
& \int_{0}^{t}\left\{\left(\boldsymbol{w}_{1}, \partial_{t} A_{\mathrm{h}} \boldsymbol{w}_{\varepsilon}\right)-\nu\left(\nabla \boldsymbol{w}_{1}, \nabla A_{\mathrm{h}} \boldsymbol{w}_{\varepsilon}\right)+\left(\boldsymbol{w}_{1} \otimes \boldsymbol{w}_{1}, \nabla \boldsymbol{w}_{\varepsilon}\right)\right\}(s) \mathrm{d} s \\
& =\int_{0}^{t}\left(A_{\mathrm{h}}^{\frac{1}{2}} \rho_{1} \boldsymbol{e}_{3}, A_{\mathrm{h}}^{\frac{1}{2}} \boldsymbol{w}_{\varepsilon}\right)(s) \mathrm{d} s+\left(\boldsymbol{w}_{1}(t), A_{\mathrm{h}} \boldsymbol{w}_{\varepsilon}(t)\right)-\left(\boldsymbol{w}_{1}(0), A_{\mathrm{h}} \boldsymbol{w}_{\varepsilon}(0)\right),
\end{aligned}
$$

and hence

$$
\begin{aligned}
& \int_{0}^{t}\left\{\left(\boldsymbol{w}_{1}, \partial_{t} \boldsymbol{w}_{\varepsilon}\right)+\alpha^{2}\left(\nabla_{\mathrm{h}} \boldsymbol{w}_{1}, \partial_{t} \nabla_{\mathrm{h}} \boldsymbol{w}_{\varepsilon}\right)-\nu\left(\nabla \boldsymbol{w}_{1}, \nabla \boldsymbol{w}_{\varepsilon}\right)\right. \\
& \left.\quad-\nu \alpha^{2}\left(\nabla_{\mathrm{h}} \nabla \boldsymbol{w}_{1}, \nabla_{\mathrm{h}} \nabla \boldsymbol{w}_{\varepsilon}\right)+\left(\left(\boldsymbol{w}_{1} \cdot \nabla\right) \boldsymbol{w}_{\varepsilon}, \boldsymbol{w}_{1}\right)\right\}(s) \mathrm{d} s \\
& =\int_{0}^{t}\left(A_{\mathrm{h}}^{\frac{1}{2}} \rho_{1} \boldsymbol{e}_{3}, A_{\mathrm{h}}^{\frac{1}{2}} \boldsymbol{w}_{\varepsilon}\right)(s) \mathrm{d} s+\left(\boldsymbol{w}_{1}(t), \boldsymbol{w}_{\varepsilon}(t)\right)+\alpha^{2}\left(\nabla_{\mathrm{h}} \boldsymbol{w}_{1}(t), \nabla_{\mathrm{h}} \boldsymbol{w}_{\varepsilon}(t)\right) \\
& \quad-\left(\boldsymbol{w}_{1}(0), \boldsymbol{w}_{\varepsilon}(0)\right)-\alpha^{2}\left(\nabla_{\mathrm{h}} \boldsymbol{w}_{1}(0), \nabla_{\mathrm{h}} \boldsymbol{w}_{\varepsilon}(0)\right)
\end{aligned}
$$

If we take this equation and subtract the analogous one for $\boldsymbol{w}_{2}$, we deduce

$$
\begin{aligned}
& \int_{0}^{t}\left\{\left(\boldsymbol{w}, \partial_{t} \boldsymbol{w}_{\varepsilon}\right)+\alpha^{2}\left(\nabla_{\mathrm{h}} \boldsymbol{w}, \partial_{t} \nabla_{\mathrm{h}} \boldsymbol{w}_{\varepsilon}\right)-\nu\left(\nabla \boldsymbol{w}, \nabla \boldsymbol{w}_{\varepsilon}\right)-\nu \alpha^{2}\left(\nabla_{\mathrm{h}} \nabla \boldsymbol{w}, \nabla_{\mathrm{h}} \nabla \boldsymbol{w}_{\varepsilon}\right)\right. \\
& \left.\quad+\left(\left(\boldsymbol{w}_{1} \cdot \nabla\right) \boldsymbol{w}_{\varepsilon}, \boldsymbol{w}_{1}\right)-\left(\left(\boldsymbol{w}_{2} \cdot \nabla\right) \boldsymbol{w}_{\varepsilon}, \boldsymbol{w}_{2}\right)\right\}(s) \mathrm{d} s
\end{aligned}
$$




$$
\begin{aligned}
= & \int_{0}^{t}\left(A_{\mathrm{h}}^{\frac{1}{2}} \rho \boldsymbol{e}_{3}, A_{\mathrm{h}}^{\frac{1}{2}} \boldsymbol{w}_{\varepsilon}\right)(s) \mathrm{d} s+\left(\boldsymbol{w}(t), \boldsymbol{w}_{\varepsilon}(t)\right)+\alpha^{2}\left(\nabla_{\mathrm{h}} \boldsymbol{w}(t), \nabla_{\mathrm{h}} \boldsymbol{w}_{\varepsilon}(t)\right) \\
& -\left(\boldsymbol{w}(0), \boldsymbol{w}_{\varepsilon}(0)\right)-\alpha^{2}\left(\nabla_{\mathrm{h}} \boldsymbol{w}(0), \nabla_{\mathrm{h}} \boldsymbol{w}_{\varepsilon}(0)\right)
\end{aligned}
$$

since $\boldsymbol{w}(0)=\mathbf{0}$ and $\left(\left(\boldsymbol{w}_{1} \cdot \nabla\right) \boldsymbol{w}_{\varepsilon}, \boldsymbol{w}_{1}\right)-\left(\left(\boldsymbol{w}_{2} \cdot \nabla\right) \boldsymbol{w}_{\varepsilon}, \boldsymbol{w}_{2}\right)=\left((\boldsymbol{w} \cdot \nabla) \boldsymbol{w}_{\varepsilon}, \boldsymbol{w}\right)+$ $\left(\left(\boldsymbol{w}_{2} \cdot \nabla\right) \boldsymbol{w}_{\varepsilon}, \boldsymbol{w}\right)+\left((\boldsymbol{w} \cdot \nabla) \boldsymbol{w}_{\varepsilon}, \boldsymbol{w}_{2}\right)$, we obtain

$$
\begin{aligned}
& \int_{0}^{t}\left\{\left(\boldsymbol{w}, \partial_{t} \boldsymbol{w}_{\varepsilon}\right)+\alpha^{2}\left(\nabla_{\mathrm{h}} \boldsymbol{w}, \partial_{t} \nabla_{\mathrm{h}} \boldsymbol{w}_{\varepsilon}\right)-\nu\left(\nabla \boldsymbol{w}, \nabla \boldsymbol{w}_{\varepsilon}\right)-\nu \alpha^{2}\left(\nabla_{\mathrm{h}} \nabla \boldsymbol{w}, \nabla_{\mathrm{h}} \nabla \boldsymbol{w}_{\varepsilon}\right)\right. \\
& \left.\quad+\left((\boldsymbol{w} \cdot \nabla) \boldsymbol{w}_{\varepsilon}, \boldsymbol{w}\right)+\left(\left(\boldsymbol{w}_{2} \cdot \nabla\right) \boldsymbol{w}_{\varepsilon}, \boldsymbol{w}\right)+\left((\boldsymbol{w} \cdot \nabla) \boldsymbol{w}_{\varepsilon}, \boldsymbol{w}_{2}\right)\right\}(s) \mathrm{d} s \\
& =\int_{0}^{t}\left(A_{\mathrm{h}}^{\frac{1}{2}} \rho \boldsymbol{e}_{3}, A_{\mathrm{h}}^{\frac{1}{2}} \boldsymbol{w}_{\varepsilon}\right)(s) \mathrm{d} s+\left(\boldsymbol{w}(t), \boldsymbol{w}_{\varepsilon}(t)\right)+\alpha^{2}\left(\nabla_{\mathrm{h}} \boldsymbol{w}(t), \nabla_{\mathrm{h}} \boldsymbol{w}_{\varepsilon}(t)\right)
\end{aligned}
$$

Now, the equation for $\rho_{1}$ gives

$$
\begin{aligned}
& \int_{0}^{t}\left\{\left(\rho_{1}, \partial_{t} A_{\mathrm{h}} \rho_{\varepsilon}\right)+\left(\rho_{1} \boldsymbol{w}_{1}, \nabla \rho_{\varepsilon}\right)-K\left(\nabla \rho_{1}, \nabla A_{\mathrm{h}} \rho_{\varepsilon}\right)\right\}(s) \mathrm{d} s \\
& =\left(\rho_{1}(t), A_{\mathrm{h}} \rho_{\varepsilon}(t)\right)-\left(\rho_{1}(0), A_{\mathrm{h}} \rho_{\varepsilon}(0)\right) .
\end{aligned}
$$

By subtracting the analogous equation for $\rho_{2}$, we get

$$
\begin{aligned}
& \int_{0}^{t}\left\{\left(\rho, \partial_{t} A_{\mathrm{h}} \rho_{\varepsilon}\right)+\left(\rho_{1} \boldsymbol{w}_{1}, \nabla \rho_{\varepsilon}\right)-\left(\rho_{2} \boldsymbol{w}_{2}, \nabla \rho_{\varepsilon}\right)-K\left(\nabla \rho, \nabla A_{\mathrm{h}} \rho_{\varepsilon}\right)\right\}(s) \mathrm{d} s \\
& =\left(\rho(t), A_{\mathrm{h}} \rho_{\varepsilon}(t)\right)-\left(\rho(0), A_{\mathrm{h}} \rho_{\varepsilon}(0)\right) .
\end{aligned}
$$

Recalling that $\rho(0)=0$ and using

$$
\left(\rho_{1} \boldsymbol{w}_{1}, \nabla \rho_{\varepsilon}\right)-\left(\rho_{2} \boldsymbol{w}_{2}, \nabla \rho_{\varepsilon}\right)=\left(\rho \boldsymbol{w}, \nabla \rho_{\varepsilon}\right)+\left(\rho \boldsymbol{w}_{2}, \nabla \rho_{\varepsilon}\right)+\left(\rho_{2} \boldsymbol{w}, \nabla \rho_{\varepsilon}\right),
$$

we obtain

$$
\begin{aligned}
& \int_{0}^{t}\left\{\left(\rho, \partial_{t} A_{\mathrm{h}} \rho_{\varepsilon}\right)+\left(\rho \boldsymbol{w}, \nabla \rho_{\varepsilon}\right)+\left(\rho \boldsymbol{w}_{2}, \nabla \rho_{\varepsilon}\right)+\left(\rho_{2} \boldsymbol{w}, \nabla \rho_{\varepsilon}\right)-K\left(\nabla \rho, \nabla A_{\mathrm{h}} \rho_{\varepsilon}\right)\right\}(s) \mathrm{d} s \\
& =\left(\rho(t), A_{\mathrm{h}} \rho_{\varepsilon}(t)\right),
\end{aligned}
$$

i.e.

$$
\begin{aligned}
& \int_{0}^{t}\left\{\left(\rho, \partial_{t} \rho_{\varepsilon}\right)+\alpha^{2}\left(\nabla_{\mathrm{h}} \rho, \partial_{t} \nabla_{\mathrm{h}} \rho_{\varepsilon}\right)+\left(\rho \boldsymbol{w}, \nabla \rho_{\varepsilon}\right)+\left(\rho \boldsymbol{w}_{2}, \nabla \rho_{\varepsilon}\right)+\left(\rho_{2} \boldsymbol{w}, \nabla \rho_{\varepsilon}\right)\right. \\
& \left.\quad-K\left(\nabla \rho, \nabla \rho_{\varepsilon}\right)-K \alpha^{2}\left(\nabla_{\mathrm{h}} \nabla \rho, \nabla_{\mathrm{h}} \nabla \rho_{\varepsilon}\right)\right\}(s) \mathrm{d} s \\
& =\left(\rho(t), \rho_{\varepsilon}(t)\right)+\alpha^{2}\left(\nabla_{\mathrm{h}} \rho(t), \nabla_{\mathrm{h}} \rho_{\varepsilon}(t)\right) .
\end{aligned}
$$


Summing up (13) and (14) yields

$$
\begin{aligned}
& \int_{0}^{t}\left\{\left(\boldsymbol{w}, \partial_{t} \boldsymbol{w}_{\varepsilon}\right)+\alpha^{2}\left(\nabla_{\mathrm{h}} \boldsymbol{w}, \partial_{t} \nabla_{\mathrm{h}} \boldsymbol{w}_{\varepsilon}\right)+\left(\rho, \partial_{t} \rho_{\varepsilon}\right)+\alpha^{2}\left(\nabla_{\mathrm{h}} \rho, \partial_{t} \nabla_{\mathrm{h}} \rho_{\varepsilon}\right)-\nu\left(\nabla \boldsymbol{w}, \nabla \boldsymbol{w}_{\varepsilon}\right)\right. \\
& \quad-\nu \alpha^{2}\left(\nabla_{\mathrm{h}} \nabla \boldsymbol{w}, \nabla_{\mathrm{h}} \nabla \boldsymbol{w}_{\varepsilon}\right)-K\left(\nabla \rho, \nabla \rho_{\varepsilon}\right)-K \alpha^{2}\left(\nabla_{\mathrm{h}} \nabla \rho, \nabla_{\mathrm{h}} \nabla \rho_{\varepsilon}\right) \\
& \quad+\left((\boldsymbol{w} \cdot \nabla) \boldsymbol{w}_{\varepsilon}, \boldsymbol{w}\right)+\left(\left(\boldsymbol{w}_{2} \cdot \nabla\right) \boldsymbol{w}_{\varepsilon}, \boldsymbol{w}\right)+\left((\boldsymbol{w} \cdot \nabla) \boldsymbol{w}_{\varepsilon}, \boldsymbol{w}_{2}\right) \\
& \left.\quad+\left(\rho \boldsymbol{w}, \nabla \rho_{\varepsilon}\right)+\left(\rho \boldsymbol{w}_{2}, \nabla \rho_{\varepsilon}\right)+\left(\rho_{2} \boldsymbol{w}, \nabla \rho_{\varepsilon}\right)\right\}(s) \mathrm{d} s \\
& =\int_{0}^{t}\left(A_{\mathrm{h}}^{\frac{1}{2}} \rho \boldsymbol{e}_{3}, A_{\mathrm{h}}^{\frac{1}{2}} \boldsymbol{w}_{\varepsilon}\right)(s) \mathrm{d} s+\left(\boldsymbol{w}(t), \boldsymbol{w}_{\varepsilon}(t)\right)+\alpha^{2}\left(\nabla_{\mathrm{h}} \boldsymbol{w}(t), \nabla_{\mathrm{h}} \boldsymbol{w}_{\varepsilon}(t)\right) \\
& \quad+\left(\rho(t), \rho_{\varepsilon}(t)\right)+\alpha^{2}\left(\nabla_{\mathrm{h}} \rho(t), \nabla_{\mathrm{h}} \rho_{\varepsilon}(t)\right) .
\end{aligned}
$$

In order to take the limit as $\varepsilon \rightarrow 0$, we observe that, by proceeding as in the previous subsection (we can consider the integral over $\left[t_{0}, t_{1}\right]$ and then take $t_{0} \rightarrow 0, t_{1}=t$ ), we have:

$$
\begin{aligned}
& \int_{0}^{t}\left\{\left(\boldsymbol{w}, \partial_{t} \boldsymbol{w}_{\varepsilon}\right)+\alpha^{2}\left(\nabla_{\mathrm{h}} \boldsymbol{w}, \partial_{t} \nabla_{\mathrm{h}} \boldsymbol{w}_{\varepsilon}\right)+\left(\rho, \partial_{t} \rho_{\varepsilon}\right)+\alpha^{2}\left(\nabla_{\mathrm{h}} \rho, \partial_{t} \nabla_{\mathrm{h}} \rho_{\varepsilon}\right)\right\}(s) \mathrm{d} s=0, \\
& \lim _{\varepsilon \rightarrow 0} \int_{0}^{t}\left\{-\nu\left(\nabla \boldsymbol{w}, \nabla \boldsymbol{w}_{\varepsilon}\right)-\nu \alpha^{2}\left(\nabla_{\mathrm{h}} \nabla \boldsymbol{w}, \nabla_{\mathrm{h}} \nabla \boldsymbol{w}_{\varepsilon}\right)\right. \\
& \left.\quad-K\left(\nabla \rho, \nabla \rho_{\varepsilon}\right)-K \alpha^{2}\left(\nabla_{\mathrm{h}} \nabla \rho, \nabla_{\mathrm{h}} \nabla \rho_{\varepsilon}\right)\right\}(s) \mathrm{d} s \\
& =-\nu \int_{0}^{t}\left\{\|\nabla \boldsymbol{w}(s)\|^{2}+\alpha^{2}\left\|\nabla_{\mathrm{h}} \nabla \boldsymbol{w}(s)\right\|^{2}\right\} \mathrm{d} s \\
& \quad-K \int_{0}^{t}\left\{\|\nabla \rho(s)\|^{2}+\alpha^{2}\left\|\nabla_{\mathrm{h}} \nabla \rho(s)\right\|^{2}\right\} \mathrm{d} s, \\
& \lim _{\varepsilon \rightarrow 0} \int_{0}^{t}\left\{\left((\boldsymbol{w} \cdot \nabla) \boldsymbol{w}_{\varepsilon}, \boldsymbol{w}\right)+\left(\left(\boldsymbol{w}_{2} \cdot \nabla\right) \boldsymbol{w}_{\varepsilon}, \boldsymbol{w}\right)+\left((\boldsymbol{w} \cdot \nabla) \boldsymbol{w}_{\varepsilon}, \boldsymbol{w}_{2}\right)\right\}(s) \mathrm{d} s \\
& =\int_{0}^{t}\left((\boldsymbol{w} \cdot \nabla) \boldsymbol{w}, \boldsymbol{w}_{2}\right)(s) \mathrm{d} s, \\
& \lim _{\varepsilon \rightarrow 0} \int_{0}^{t}\left\{\left(\rho \boldsymbol{w}, \nabla \rho_{\varepsilon}\right)+\left(\rho \boldsymbol{w}_{2}, \nabla \rho_{\varepsilon}\right)+\left(\rho_{2} \boldsymbol{w}, \nabla \rho_{\varepsilon}\right)\right\}(s) \mathrm{d} s=\int_{0}^{t}\left(\rho_{2} \boldsymbol{w}, \nabla \rho\right)(s) \mathrm{d} s, \\
& \lim _{\varepsilon \rightarrow 0} \int_{0}^{t}\left(A_{\mathrm{h}}^{\frac{1}{2}} \rho \boldsymbol{e}_{3}, A_{\mathrm{h}}^{\frac{1}{2}} \boldsymbol{w}_{\varepsilon}\right)(s) \mathrm{d} s=\int_{0}^{t}\left(A_{\mathrm{h}}^{\frac{1}{2}} \rho(s) \boldsymbol{e}_{3}, A_{\mathrm{h}}^{\frac{1}{2}} \boldsymbol{w}(s)\right) \mathrm{d} s
\end{aligned}
$$

and also

$$
\begin{aligned}
& \left(\boldsymbol{w}(t), \boldsymbol{w}_{\varepsilon}(t)\right)+\alpha^{2}\left(\nabla_{\mathrm{h}} \boldsymbol{w}(t), \nabla_{\mathrm{h}} \boldsymbol{w}_{\varepsilon}(t)\right)+\left(\rho(t), \rho_{\varepsilon}(t)\right)+\alpha^{2}\left(\nabla_{\mathrm{h}} \rho(t), \nabla_{\mathrm{h}} \rho_{\varepsilon}(t)\right) \\
& =\frac{1}{2}\left\{\|\boldsymbol{w}(t)\|^{2}+\alpha^{2}\left\|\nabla_{\mathrm{h}} \boldsymbol{w}(t)\right\|^{2}+\|\rho(t)\|^{2}+\alpha^{2}\left\|\nabla_{\mathrm{h}} \rho(t)\right\|^{2}\right\}+O(\varepsilon) .
\end{aligned}
$$


Thus, we get

$$
\begin{aligned}
- & \nu \int_{0}^{t}\left\{\|\nabla \boldsymbol{w}(s)\|^{2}+\alpha^{2}\left\|\nabla_{\mathrm{h}} \nabla \boldsymbol{w}(s)\right\|^{2}\right\} \mathrm{d} s-K \int_{0}^{t}\left\{\|\nabla \rho(s)\|^{2}+\alpha^{2}\left\|\nabla_{\mathrm{h}} \nabla \rho(s)\right\|^{2}\right\} \mathrm{d} s \\
& +\int_{0}^{t}\left\{\left((\boldsymbol{w} \cdot \nabla) \boldsymbol{w}, \boldsymbol{w}_{2}\right)+\left(\rho_{2} \boldsymbol{w}, \nabla \rho\right)\right\}(s) \mathrm{d} s \\
= & \int_{0}^{t}\left(A_{\mathrm{h}}^{\frac{1}{2}} \rho(s) \boldsymbol{e}_{3}, A_{\mathrm{h}}^{\frac{1}{2}} \boldsymbol{w}(s)\right) \mathrm{d} s \\
& +\frac{1}{2}\left\{\|\boldsymbol{w}(t)\|^{2}+\alpha^{2}\left\|\nabla_{\mathrm{h}} \boldsymbol{w}(t)\right\|^{2}+\|\rho(t)\|^{2}+\alpha^{2}\left\|\nabla_{\mathrm{h}} \rho(t)\right\|^{2}\right\},
\end{aligned}
$$

and hence

$$
\begin{aligned}
& \frac{1}{2}\left\{\|\boldsymbol{w}(t)\|^{2}+\alpha^{2}\left\|\nabla_{\mathrm{h}} \boldsymbol{w}(t)\right\|^{2}+\|\rho(t)\|^{2}+\alpha^{2}\left\|\nabla_{\mathrm{h}} \rho(t)\right\|^{2}\right\} \\
& \quad+\nu \int_{0}^{t}\left\{\|\nabla \boldsymbol{w}(s)\|^{2}+\alpha^{2}\left\|\nabla_{\mathrm{h}} \nabla \boldsymbol{w}(s)\right\|^{2}\right\} \mathrm{d} s \\
& \quad+K \int_{0}^{t}\left\{\|\nabla \rho(s)\|^{2}+\alpha^{2}\left\|\nabla_{\mathrm{h}} \nabla \rho(s)\right\|^{2}\right\} \mathrm{d} s \\
& \leq \frac{1}{2} \int_{0}^{t}\left\{\|\boldsymbol{w}(s)\|^{2}+\alpha^{2}\left\|\nabla_{\mathrm{h}} \boldsymbol{w}(s)\right\|^{2}+\|\rho(s)\|^{2}+\alpha^{2}\left\|\nabla_{\mathrm{h}} \rho(s)\right\|^{2}\right\} \mathrm{d} s \\
& \quad+\int_{0}^{t}\left|\left((\boldsymbol{w} \cdot \nabla) \boldsymbol{w}, \boldsymbol{w}_{2}\right)+\left(\rho_{2} \boldsymbol{w}, \nabla \rho\right)\right|(s) \mathrm{d} s .
\end{aligned}
$$

The term $\left((\boldsymbol{w} \cdot \nabla) \boldsymbol{w}, \boldsymbol{w}_{2}\right)$ can be estimated as follows:

$$
\begin{aligned}
& \int_{0}^{t}\left|\left((\boldsymbol{w}(s) \cdot \nabla) \boldsymbol{w}(s), \boldsymbol{w}_{2}(s)\right)\right| \mathrm{d} s \\
& \leq \int_{0}^{t}\left\{\frac{\nu}{2}\|\nabla \boldsymbol{w}(s)\|^{2}+\frac{\nu \alpha^{2}}{2}\left\|\nabla_{\mathrm{h}} \nabla \boldsymbol{w}(s)\right\|^{2}\right\} \mathrm{d} s \\
& \quad+C \int_{0}^{t}\left\{\frac{1}{\nu^{3}}\left\|\nabla_{\mathrm{h}} \boldsymbol{w}_{2}(s)\right\|^{4}+\frac{1+\alpha^{2}}{\nu \alpha}\left\|\nabla \boldsymbol{w}_{2}(s)\right\|^{2}\right\}\|\boldsymbol{w}(s)\|^{2} \mathrm{~d} s .
\end{aligned}
$$

In order to estimate $\left|\left(\rho_{2} \boldsymbol{w}, \nabla \rho\right)\right|$, we utilize the identity

$$
\left(\rho_{2} \boldsymbol{w}, \nabla \rho\right)=\left(\rho_{2} \boldsymbol{w}^{\mathrm{h}}, \nabla_{\mathrm{h}} \rho\right)+\left(\rho_{2} w^{3}, \partial_{3} \rho\right)
$$

and the following estimates, where we use Hölder, Gagliardo-Nirenberg and Young inequalities. For the first term in the right-hand side, we have

$$
\begin{aligned}
\left|\left(\rho_{2} \boldsymbol{w}^{\mathrm{h}}, \nabla_{\mathrm{h}} \rho\right)\right| & \leq\left\|\rho_{2}\right\|_{L^{3}}\|\boldsymbol{w}\|\left\|\nabla_{\mathrm{h}} \rho\right\|_{L^{6}} \\
& \leq C\left\|\rho_{2}\right\|^{\frac{1}{2}}\left\|\nabla \rho_{2}\right\|^{\frac{1}{2}}\|\boldsymbol{w}\|\left\|\nabla_{\mathrm{h}} \nabla \rho\right\| \\
& \leq \frac{K \alpha^{2}}{2}\left\|\nabla_{\mathrm{h}} \nabla \rho\right\|^{2}+\frac{C}{K \alpha^{2}}\left\|\rho_{2}\right\|\left\|\nabla \rho_{2}\right\|\|\boldsymbol{w}\|^{2} \\
& \leq \frac{K \alpha^{2}}{2}\left\|\nabla_{\mathrm{h}} \nabla \rho\right\|^{2}+\frac{C}{K \alpha^{2}}\left(\left\|\rho_{2}\right\|^{2}+\left\|\nabla \rho_{2}\right\|^{2}\right)\|\boldsymbol{w}\|^{2} .
\end{aligned}
$$


For the second term, we have

$$
\begin{aligned}
\left|\left(\rho_{2} w^{3}, \partial_{3} \rho\right)\right| & \leq\left\|\rho_{2}\right\|_{L^{3}}\left\|w^{3}\right\|_{L^{6}}\left\|\partial_{3} \rho\right\| \\
& \leq C\left\|\rho_{2}\right\|^{\frac{1}{2}}\left\|\nabla \rho_{2}\right\|^{\frac{1}{2}}\left\|\nabla w^{3}\right\|\|\nabla \rho\| \\
& \leq \frac{K}{2}\|\nabla \rho\|^{2}+\frac{C}{K}\left\|\rho_{2}\right\|\left\|\nabla \rho_{2}\right\|\left\|\nabla w^{3}\right\|^{2} \\
& \leq \frac{K}{2}\|\nabla \rho\|^{2}+\frac{C}{K \alpha^{2}}\left(\left\|\rho_{2}\right\|^{2}+\left\|\nabla \rho_{2}\right\|^{2}\right) \alpha^{2}\left\|\nabla_{\mathrm{h}} \boldsymbol{w}\right\|^{2},
\end{aligned}
$$

where we have used again the fact that, since $\nabla \cdot \boldsymbol{w}=0$, there holds $\partial_{3} w^{3}=-\nabla_{\mathrm{h}} \cdot \boldsymbol{w}^{\mathrm{h}}$, and consequently

$$
\left\|\nabla w^{3}\right\|^{2}=\left\|\nabla_{\mathrm{h}} w^{3}\right\|^{2}+\left\|\partial_{3} w^{3}\right\|^{2}=\left\|\nabla_{\mathrm{h}} w^{3}\right\|^{2}+\left\|\nabla_{\mathrm{h}} \cdot \boldsymbol{w}^{\mathrm{h}}\right\|^{2} \leq 2\left\|\nabla_{\mathrm{h}} \boldsymbol{w}\right\|^{2} .
$$

By inserting (16), (17) in (15), and setting

$$
\begin{aligned}
& Y(t):=\|\boldsymbol{w}(t)\|^{2}+\alpha^{2}\left\|\nabla_{\mathrm{h}} \boldsymbol{w}(t)\right\|^{2}+\|\rho(t)\|^{2}+\alpha^{2}\left\|\nabla_{\mathrm{h}} \rho(t)\right\|^{2}, \\
& B(s):=\frac{1}{\nu^{3}}\left\|\nabla_{\mathrm{h}} \boldsymbol{w}_{2}(s)\right\|^{4}+\frac{1+\alpha^{2}}{\nu \alpha}\left\|\nabla \boldsymbol{w}_{2}(s)\right\|^{2}+\frac{1}{K \alpha^{2}}\left(\left\|\rho_{2}(s)\right\|^{2}+\left\|\nabla \rho_{2}(s)\right\|^{2}\right),
\end{aligned}
$$

we obtain

$$
\begin{aligned}
& Y(t)+\nu \int_{0}^{t}\left\{\|\nabla \boldsymbol{w}(s)\|^{2}+\alpha^{2}\left\|\nabla_{\mathrm{h}} \nabla \boldsymbol{w}(s)\right\|^{2}\right\} \mathrm{d} s+K \int_{0}^{t}\left\{\|\nabla \rho(s)\|^{2}+\alpha^{2}\left\|\nabla_{\mathrm{h}} \nabla \rho(s)\right\|^{2}\right\} \mathrm{d} s \\
& \leq \int_{0}^{t}(1+C B(s)) Y(s) \mathrm{d} s .
\end{aligned}
$$

Since $B \in L^{1}(0, T)$, an application of the Gronwall lemma implies $Y(t) \equiv 0$, i.e. uniqueness of the solution and also, by adapting the argument, continuous dependence on the data.

\section{Other models with horizontal filtering}

In this section we briefly study other two models in which the equation for the density has not been filtered. In this section we just sketch the proofs since they are along the mainstream of those given with great details in the previous part of the paper.

It is interesting to observe that the analysis of the following two models is motivated by the fact that the numerical simulations in $[24,25]$ seem to justify that the regularization of the equation for the density is not necessary to have a well-posed system. This has been later rigorously proved or reviewed in [8], but the results obtained there do not cover the possible regularization only in the two horizontal variables (this is similar to what happens in magnetohydrodynamics, where filtering the equation for the magnetic field can be avoided, see $[12,13]$ ). Hence, here we take a closer look to these models. In particular, we will not reproduce all the calculations, but just show the changes needed to deal with different models. 
3.1. A LES model without filtering in the equation for $\rho$. We consider now the system

$$
\begin{aligned}
\partial_{t} \boldsymbol{w}+\nabla \cdot \overline{(\boldsymbol{w} \otimes \boldsymbol{w}}^{\mathrm{h}}-\nu \Delta \boldsymbol{w}+\nabla q & =-\bar{\rho}^{\mathrm{h}} \boldsymbol{e}_{3} & & \text { in } D \times] 0, T[, \\
\nabla \cdot \boldsymbol{w} & =0 & & \text { in } D \times] 0, T[, \\
\partial_{t} \rho+\nabla \cdot\left(\rho A_{\mathrm{h}} \boldsymbol{w}\right)-K \Delta \rho & =0 & & \text { in } D \times] 0, T[,
\end{aligned}
$$

where the third equation has not been filtered, and so we have $\rho=\vartheta$. In particular, in the equation for $\rho$ the transport is made by the field $\boldsymbol{u}=A_{h} \boldsymbol{w}$. For a regular weak solution, in this case, we require the regularity

$$
\begin{aligned}
\boldsymbol{w} & \in L^{\infty}\left(0, T ; V_{\mathrm{h}}\right) \cap L^{2}\left(0, T ; V \cap\left(H_{\mathrm{h}}^{2}\right)^{3}\right) \cap C_{\mathrm{w}}\left(0, T ; V_{\mathrm{h}}\right), \\
w_{3} & \in L^{\infty}\left(0, T ; H^{1}\right) \cap L^{2}\left(0, T ; H^{2}\right), \\
\partial_{t} \boldsymbol{w} & \in L^{2}\left(0, T ; V^{*}\right), \\
\rho & \in L^{\infty}\left(0, T ; L^{2}\right) \cap L^{2}\left(0, T ; H^{1}\right) \cap C_{\mathrm{w}}\left(0, T ; L^{2}\right), \\
\partial_{t} \rho & \in L^{2}\left(0, T ; H^{-\frac{5}{2}-\delta}\right), \quad \delta>0,
\end{aligned}
$$

and that the weak formulation

$$
\begin{aligned}
& \int_{0}^{+\infty}\left\{\left(\boldsymbol{w}, \partial_{t} \boldsymbol{\varphi}\right)-\nu(\nabla \boldsymbol{w}, \nabla \boldsymbol{\varphi})+\left(\boldsymbol{w} \otimes \boldsymbol{w}, \nabla \overline{\boldsymbol{\varphi}}^{\mathrm{h}}\right)\right\}(s) \mathrm{d} s \\
& =\int_{0}^{+\infty}\left(\bar{\rho}^{\mathrm{h}} \boldsymbol{e}_{3}, \boldsymbol{\varphi}\right)(s) \mathrm{d} s-(\boldsymbol{w}(0), \boldsymbol{\varphi}(0)), \\
& \int_{0}^{+\infty}\left\{\left(\rho, \partial_{t} \psi\right)+\left(\rho A_{\mathrm{h}} \boldsymbol{w}, \nabla \psi\right)-K(\nabla \rho, \nabla \psi)\right\}(s) \mathrm{d} s \\
& =-(\rho(0), \psi(0)),
\end{aligned}
$$

is satisfied for each $\boldsymbol{\varphi} \in\left(C_{0}^{\infty}\left(D \times[0, T[))^{3}\right.\right.$ such that $\nabla \cdot \boldsymbol{\varphi}=0$, and for each $\psi \in C_{0}^{\infty}(D \times[0, T[)$.

We have the following result

Theorem 3.1. Let be given $\left(\boldsymbol{w}_{0}, \rho_{0}\right) \in V_{\mathrm{h}} \times L^{2}(D)$ and $\nu, K>0$. Then there exists (at least) a regular weak solution to (18)-(20), with $\boldsymbol{w}=\mathbf{0}$ and $\rho=0$ on ] $0, T\left[\times \Gamma\right.$, and $(\boldsymbol{w}(0, \boldsymbol{x}), \rho(0, \boldsymbol{x}))=\left(\boldsymbol{w}_{0}, \rho_{0}\right)$. Moreover, regular weak solutions satisfy the energy (of the model) inequality

$$
\begin{aligned}
& \frac{1}{2}\left(\|\boldsymbol{w}(t)\|^{2}+\alpha^{2}\left\|\nabla_{\mathrm{h}} \boldsymbol{w}(t)\right\|^{2}+\|\rho(t)\|^{2}\right) \\
& \quad+\nu \int_{0}^{t}\left(\|\nabla \boldsymbol{w}(s)\|^{2}+\alpha^{2}\left\|\nabla_{\mathrm{h}} \nabla \boldsymbol{w}(s)\right\|^{2}\right) \mathrm{d} s+K \int_{0}^{t}\|\nabla \rho(s)\|^{2} \mathrm{~d} s \\
& \leq \frac{1}{2}\left(\|\boldsymbol{w}(0)\|^{2}+\alpha^{2}\left\|\nabla_{\mathrm{h}} \boldsymbol{w}(0)\right\|^{2}+\|\rho(0)\|^{2}\right)-\int_{0}^{t}\left(\rho(s) \boldsymbol{e}_{3}, \boldsymbol{w}(s)\right) \mathrm{d} s .
\end{aligned}
$$


Proof. We can easily adapt the steps performed for the previous model, to prove existence and energy inequality. This can be obtained by noticing that in the equation (20) we have $\nabla \cdot\left(\rho A_{\mathrm{h}} \boldsymbol{w}\right)$ instead of $\nabla \cdot\left(\overline{\rho \boldsymbol{w}^{\mathrm{h}}}\right)$.

The only essential difference concerns the regularity of $\partial_{t} \rho_{m}$. We have the following estimate

$$
\begin{aligned}
\left|\left(\rho_{m}(t) A_{\mathrm{h}} \boldsymbol{w}_{m}(t), \nabla \psi(t)\right)\right| & \leq\left\|\rho_{m}(t)\right\|_{L^{2}}\left\|A_{\mathrm{h}} \boldsymbol{w}_{m}(t)\right\|_{L^{2}}\|\nabla \psi(t)\|_{L^{\infty}} \\
& \leq C\left\|\rho_{m}(t)\right\|_{L^{2}}\left\|\boldsymbol{w}_{m}(t)\right\|_{H_{\mathrm{h}}^{2}}\|\psi(t)\|_{H^{\frac{5}{2}+\delta}}
\end{aligned}
$$

and also

$$
\int_{0}^{T}\left|\left(\rho_{m}(t) \boldsymbol{w}_{m}(t), \nabla \psi(t)\right)\right| \mathrm{d} t \leq C\left\|\rho_{m}\right\|_{L^{\infty} L^{2}}\left\|\boldsymbol{w}_{m}\right\|_{L^{2} H_{\mathrm{h}}^{2}}\|\psi\|_{L^{2} H^{\frac{5}{2}+\delta}}
$$

In order to estimate $\left|\left(\nabla \rho_{m}, \nabla \psi\right)\right|$, we utilize $\rho_{m} \in L^{2}\left(0, T ; H^{1}\right)$ and show that

$$
\int_{0}^{T}\left|\left(\nabla \rho_{m}(t), \nabla \psi(t)\right)\right| \mathrm{d} t \leq\left\|\nabla \rho_{m}\right\|_{L^{2} L^{2}}\|\nabla \psi\|_{L^{2} L^{2}}
$$

so that finally $\partial_{t} \rho_{m} \in L^{2}\left(0, T ; H^{-\frac{5}{2}-\delta}\right)$. Note that, indeed, we do not strictly need $\rho_{m} \in L^{2}\left(0, T ; H^{1}\right)$, since we can use in alternative

$$
\int_{0}^{T}\left|\left(\nabla \rho_{m}(t), \nabla \psi(t)\right)\right| \mathrm{d} t \leq\left\|\rho_{m}\right\|_{L^{2} L^{2}}\|\psi\|_{L^{2} H^{2}},
$$

and the rest of the proof is almost the same.

What is relevant is that now the energy identity cannot be proved and it has difficulties similar to those known for the weak solutions to the 3D NavierStokes equations. In fact, if we try to reproduce the steps performed for the previous model, the term $\left(\rho A_{\mathrm{h}} \boldsymbol{w}, \nabla \rho_{k, \varepsilon}\right)$ appears. Here, the problem is to show that $\left(\rho A_{\mathrm{h}} \boldsymbol{w}, \nabla \rho_{k, \varepsilon}\right) \rightarrow\left(\rho A_{\mathrm{h}} \boldsymbol{w}, \nabla \rho_{\varepsilon}\right)$, as $k \rightarrow+\infty$.

We are not able to achieve uniqueness for this model as well. Without the energy equality we cannot use the same tools, but one can understand why the result cannot be deduced from the known regularity of the solution, directly by performing some formal calculations. In order to get uniqueness, we could in fact consider two solutions $\left(\boldsymbol{w}_{1}, \rho_{1}\right)$ and $\left(\boldsymbol{w}_{2}, \rho_{2}\right)$ to $(18)-(20)$ with the same initial data, and then find the equations satisfied by $(\boldsymbol{w}, \rho)$, where $\boldsymbol{w}:=\boldsymbol{w}_{1}-\boldsymbol{w}_{2}$ and $\rho:=\rho_{1}-\rho_{2}$. We have the same difficulty to pass to the limit $\left(\rho A_{\mathrm{h}} \boldsymbol{w}, \nabla \rho_{k, \varepsilon}\right) \rightarrow\left(\rho A_{\mathrm{h}} \boldsymbol{w}, \nabla \rho_{\varepsilon}\right)$, as $k \rightarrow+\infty$. Even if this convergence were true, the term

$$
\int_{0}^{t}\left(\rho_{2} A_{\mathrm{h}} \boldsymbol{w}, \nabla \rho\right)(s) \mathrm{d} s
$$

will appear, and we are not able to estimate this term in order to apply the Gronwall lemma. 
3.2. Another LES model without filtering in the equation for $\rho$. Since the previous model seems not to introduce enough regularization to have the requested properties, we can consider also the following (more regular) LES model

$$
\begin{aligned}
\partial_{t} \boldsymbol{w}+\nabla \cdot \overline{(\boldsymbol{w} \otimes \boldsymbol{w}}^{\mathrm{h}}-\nu \Delta \boldsymbol{w}+\nabla q & =-\bar{\rho}^{\mathrm{h}} \boldsymbol{e}_{3} & & \text { in } D \times] 0, T[, \\
\nabla \cdot \boldsymbol{w} & =0 & & \text { in } D \times] 0, T[, \\
\partial_{t} \rho+\nabla \cdot(\rho \boldsymbol{w})-K \Delta \rho & =0 & & \text { in } D \times] 0, T[.
\end{aligned}
$$

Once again, the third equation has not been filtered, so $\rho=\vartheta$. Nevertheless observe that, with respect to the previous model, the third equation is more regular, since the transport is now made by the field $\boldsymbol{w}$ and not by $A_{h} \boldsymbol{w}$.

For regular weak solutions we have to require the following regularity

$$
\begin{aligned}
\boldsymbol{w} & \in L^{\infty}\left(0, T ; V_{\mathrm{h}}\right) \cap L^{2}\left(0, T ; V \cap\left(H_{\mathrm{h}}^{2}\right)^{3}\right) \cap C_{\mathrm{w}}\left(0, T ; V_{\mathrm{h}}\right), \\
w_{3} & \in L^{\infty}\left(0, T ; H^{1}\right) \cap L^{2}\left(0, T ; H^{2}\right), \\
\partial_{t} \boldsymbol{w} & \in L^{2}\left(0, T ; V^{*}\right), \\
\rho & \in L^{\infty}\left(0, T ; L^{2}\right) \cap L^{2}\left(0, T ; H^{1}\right) \cap C_{\mathrm{w}}\left(0, T ; L^{2}\right), \\
\partial_{t} \rho & \in L^{2}\left(0, T ; H^{-\frac{7}{4}}\right) .
\end{aligned}
$$

Moreover, we ask that the weak formulation

$$
\begin{aligned}
& \int_{0}^{+\infty}\left\{\left(\boldsymbol{w}, \partial_{t} \boldsymbol{\varphi}\right)-\nu(\nabla \boldsymbol{w}, \nabla \boldsymbol{\varphi})+\left(\boldsymbol{w} \otimes \boldsymbol{w}, \nabla \overline{\boldsymbol{\varphi}}^{\mathrm{h}}\right)\right\}(s) \mathrm{d} s \\
& =\int_{0}^{+\infty}\left(\bar{\rho}^{\mathrm{h}} \boldsymbol{e}_{3}, \boldsymbol{\varphi}\right)(s) \mathrm{d} s-(\boldsymbol{w}(0), \boldsymbol{\varphi}(0)), \\
& \int_{0}^{+\infty}\left\{\left(\rho, \partial_{t} \psi\right)+(\rho \boldsymbol{w}, \nabla \psi)-K(\nabla \rho, \nabla \psi)\right\}(s) \mathrm{d} s \\
& =-(\rho(0), \psi(0)),
\end{aligned}
$$

is satisfied for each $\boldsymbol{\varphi} \in\left(C_{0}^{\infty}\left(D \times[0, T[))^{3}\right.\right.$ such that $\nabla \cdot \boldsymbol{\varphi}=0$, and for each $\psi \in C_{0}^{\infty}(D \times[0, T[)$. We are nevertheless able to obtain the following result.

Theorem 3.2. Let be given $\left(\boldsymbol{w}_{0}, \rho_{0}\right) \in V_{\mathrm{h}} \times L^{2}(D)$ and $\nu, K>0$. Then there exists (at least) a regular weak solution to (21)-(23), with $\boldsymbol{w}=\mathbf{0}$ and $\rho=0$ on ] $0, T\left[\times \Gamma\right.$, and $(\boldsymbol{w}(0, \boldsymbol{x}), \rho(0, \boldsymbol{x}))=\left(\boldsymbol{w}_{0}, \rho_{0}\right)$. Moreover, regular weak solutions satisfy the energy (of the model) inequality

$$
\begin{aligned}
& \frac{1}{2}\left(\|\boldsymbol{w}(t)\|^{2}+\alpha^{2}\left\|\nabla_{\mathrm{h}} \boldsymbol{w}(t)\right\|^{2}+\|\rho(t)\|^{2}\right) \\
& \quad+\nu \int_{0}^{t}\left(\|\nabla \boldsymbol{w}(s)\|^{2}+\alpha^{2}\left\|\nabla_{\mathrm{h}} \nabla \boldsymbol{w}(s)\right\|^{2}\right) \mathrm{d} s+K \int_{0}^{t}\|\nabla \rho(s)\|^{2} \mathrm{~d} s \\
& \leq \frac{1}{2}\left(\|\boldsymbol{w}(0)\|^{2}+\alpha^{2}\left\|\nabla_{\mathrm{h}} \boldsymbol{w}(0)\right\|^{2}+\|\rho(0)\|^{2}\right)-\int_{0}^{t}\left(\rho(s) \boldsymbol{e}_{3}, \boldsymbol{w}(s)\right) \mathrm{d} s .
\end{aligned}
$$


Proof. Again existence and energy inequality are identical to the ones for the first model, except for one term: in equation $(23)$ we have $\nabla \cdot(\rho \boldsymbol{w})$ instead of $\nabla \cdot\left(\rho A_{\mathrm{h}} \boldsymbol{w}\right)$.

Concerning for the regularity of $\partial_{t} \rho_{m}$, we have

$$
\begin{aligned}
\left|\left(\rho_{m}(t) \boldsymbol{w}_{m}(t), \nabla \psi(t)\right)\right| & \leq\left\|\rho_{m}(t)\right\|_{L^{2}}\left\|\boldsymbol{w}_{m}(t)\right\|_{L^{4}}\|\nabla \psi(t)\|_{L^{4}} \\
& \leq C\left\|\rho_{m}(t)\right\|_{L^{2}}\left\|\boldsymbol{w}_{m}(t)\right\|_{V}\|\psi(t)\|_{H^{\frac{7}{4}}}
\end{aligned}
$$

and

$$
\int_{0}^{T}\left|\left(\rho_{m}(t) \boldsymbol{w}_{m}(t), \nabla \psi(t)\right)\right| \mathrm{d} t \leq C\left\|\rho_{m}\right\|_{L^{\infty} L^{2}}\left\|\boldsymbol{w}_{m}\right\|_{L^{2} V}\|\psi\|_{L^{2} H^{\frac{7}{4}}}
$$

We have $\rho_{m} \in L^{2}\left(0, T ; H^{1}\right)$ and hence

$$
\int_{0}^{T}\left|\left(\nabla \rho_{m}(t), \nabla \psi(t)\right)\right| \mathrm{d} t \leq\left\|\nabla \rho_{m}\right\|_{L^{2} L^{2}}\|\nabla \psi\|_{L^{2} L^{2}}
$$

so that $\partial_{t} \rho_{m} \in L^{2}\left(0, T ; H^{-\frac{7}{4}}\right)$.

When looking to the energy identity, we are not able to prove it: if we try to proceed as for the first model, we note that in equation (23) we have $\nabla \cdot(\rho \boldsymbol{w})$ instead of $\nabla \cdot\left(\rho A_{\mathrm{h}} \boldsymbol{w}\right)$. Here the problem is to show that $\left(\rho \boldsymbol{w}, \nabla \rho_{k, \varepsilon}\right) \rightarrow$ $\left(\rho \boldsymbol{w}, \nabla \rho_{\varepsilon}\right)$, as $k \rightarrow+\infty$.

Also in this case the uniqueness is not achieved since we have the same difficulty to pass to the limit $\left(\rho \boldsymbol{w}, \nabla \rho_{k, \varepsilon}\right) \rightarrow\left(\rho \boldsymbol{w}, \nabla \rho_{\varepsilon}\right)$ as $k \rightarrow+\infty$. Even if this convergence were, the term

$$
\int_{0}^{t}\left(\rho_{2} \boldsymbol{w}, \nabla \rho\right)(s) \mathrm{d} s
$$

will appear, and we are not able to estimate this term in order to apply the Gronwall lemma.

Acknowledgement. Both authors are members of the GNAMPA, of the Istituto Nazionale di Alta Matematica "F. Severi".

\section{References}

[1] Berselli, L. C., Analysis of a large eddy simulation model based on anisotropic filtering. J. Math. Anal. Appl. 386 (2012), $149-170$.

[2] Berselli, L. C., Catania, D. and Lewandowski R., Convergence of approximate deconvolution models to the mean magnetohydrodynamics equations: analysis of two models. J. Math. Anal. Appl. 401 (2013), $864-880$. 
[3] Berselli, L. C., Fischer, P., Iliescu, T. and Özgökmen, T., Horizontal large eddy simulation of stratified mixing in a lock-exchange system. J. Sci. Comput. 49 (2011), $3-20$.

[4] Berselli, L. C., Galdi, G. P., Iliescu, T. and Layton, W. J., Mathematical analysis for the rational large eddy simulation model. Math. Models Methods Appl. Sci. 12 (2002), $1131-1152$.

[5] Berselli, L. C., Grisanti, C. R. and John, V., Analysis of commutation errors for functions with low regularity. J. Comput. Appl. Math. 206 (2007), 1027 - 1045.

[6] Berselli, L. C., Iliescu, T. and Layton, W. J., Mathematics of Large Eddy Simulation of Turbulent Flows. Sci. Comput. Berlin: Springer 2006.

[7] Berselli, L. C. and Lewandowski, R., Convergence of approximate deconvolution models to the mean Navier-Stokes equations. Ann. Inst. H. Poincaré Anal. Non Linéaire 29 (2012), 171 - 198.

[8] Berselli, L. C. and Spirito, S., On the Boussinesq system: regularity criteria and singular limits. Methods Appl. Anal. 18 (2011), 391 - 416.

[9] Bisconti, L., On the convergence of an approximate deconvolution model to the 3D mean Boussinesq equations. Math. Methods Appl. Sci. (2014) (available online first doi: 10.1002/mma.3160).

[10] Cao, C. and Titi, E. S., Global well-posedness of the three-dimensional viscous primitive equations of large scale ocean and atmosphere dynamics. Ann. Math. (2), 166 (2007), 245 - 267.

[11] Cao, C., Titi, E. S. and Ziane, M., A "horizontal" hyper-diffusion threedimensional thermocline planetary geostrophic model: well-posedness and long-time behaviour. Nonlinearity 17 (2004), 1749 - 1776.

[12] Catania, D. and Secchi, P., Global existence and finite dimensional global attractor for a 3D double viscous MHD- $\alpha$ model. Commun. Math. Sci. 8 (2010), $1021-1040$.

[13] Catania, D. and Secchi, P., Global existence for two regularized MHD models in three space-dimension. Portugal. Math. (N.S.) 68 (2011), $41-52$.

[14] Cerminara, M., Berselli, L. C., Esposti Ongaro, T. and Salvetti, M. V., Direct numerical simulation of a compressible multiphase flow through the Eulerian approach. In: Direct and Large-Eddy Simulation IX. (eds.: J. Fröhlich et al.). ERCOFTAC Ser. 20. Berlin: Springer 2015.

[15] Chemin, J.-Y., Desjardins, B., Gallagher, I. and Grenier, E., Fluids with anisotropic viscosity, M2AN Math. Model. Numer. Anal. 34 (2000), 315 - 335.

[16] Constantin, P. and Foias, C., Navier-Stokes Equations. Chicago Lect. Math. Chicago (IL): Univ. Chicago Press 1988.

[17] Cushman-Roisin, B. and Beckers, J.-M., Introduction to Geophysical Fluid Dynamics. Second edition. Internat. Geophysics 101. Waltham (MA): Academic Press 2011. 
[18] Dunca, A., John, V. and Layton, W. J., The commutation error of the space averaged Navier-Stokes equations on a bounded domain. In: Contributions to Current Challenges in Mathematical Fluid Mechanics (eds.: G. P. Galdi et al.). Adv. Math. Fluid Mech. Basel: Birkhäuser 2004, pp. 53 - 78.

[19] Foias, C., Manley, O., Rosa, R. and Temam R., Navier-Stokes Equations and Turbulence. Encyclopedia Math. Appl. 83. Cambridge: Cambridge Univ. Press 2001.

[20] Galdi, G. P., An introduction to the Navier-Stokes initial-boundary value problem. In: Fundamental Directions in Mathematical Fluid Mechanics (eds.: G. P. Galdi et al.). Adv. Math. Fluid Mech. Basel: Birkhäuser 2000, pp. $1-70$.

[21] Geurts B. J., Elements of Direct and Large Eddy Simulation. Flourtown (PA): Edwards Publishing 2003.

[22] Layton W. J. and Lewandowski, R., On a well-posed turbulence model. Discrete Contin. Dyn. Syst. Ser. B 6 (2006), $111-128$.

[23] Majda, A. J., Introduction to PDEs and Waves for the Atmosphere and Ocean. Courant Lect. Notes Math. 9. New York: New York Univ. 2003.

[24] Özgökmen, T., Iliescu, T. and Fischer, P., Large eddy simulation of stratified mixing in a three-dimensional lock-exchange system. Ocean Modelling 26 (2009), $134-155$.

[25] Özgökmen, T., Iliescu, T., Fischer, P., Srinivasan, A. and Duan, J., Large eddy simulation of stratified mixing in two-dimensional dam-break problem in a rectangular enclosed domain. Ocean Modelling 16 (2007), 106 - 140.

[26] Rajagopal, K. R., Růžička, M. and Srinivasa, A. R., On the OberbeckBoussinesq approximation. Math. Models Methods Appl. Sci. 6 (1996), 1157 - 1167.

[27] Rummler, B., The eigenfunctions of the Stokes operator in special domains. II. Z. Angew. Math. Mech. 77 (1997), $669-675$.

[28] Sagaut, P., Large Eddy Simulation for Incompressible Flows. An introduction (with an introduction by M. Lesieur, translated from the 1998 French original by the author). Sci. Comput. Berlin: Springer 2001.

[29] Stolz, S., Adams, N. A. and Kleiser, L., An approximate deconvolution model for large-eddy simulation with application to incompressible wall-bounded flows. Phys. Fluids 13 (2001), $997-1015$.

Received November 14, 2013 